\title{
Peter Stemmer*
}

\section{Moral, moralisches Müssen und Sanktionen}

\author{
Eine Retraktation
}

DOI 10.1515/dzph-2017-0045

\begin{abstract}
The primary aim of this article is to respond to recent discussion on the sanction theory of moral normativity. I have developed such a view in several works, and in face of the strong criticism, I want to reexamine the issue and discuss five main objections. The central question will be how one can make sense of the moral must.
\end{abstract}

Keywords: moral normativity, categorical imperatives, desire-dependence, artificial reasons, social sanctions, moral motivation

Eine elementare Schwäche der gegenwärtigen Moralphilosophie liegt darin, dass in ihren Diskussionen unterschiedliche Vorstellungen davon, was eine Moral ist, eine wesentliche Rolle spielen und den Hintergrund für tiefgehende Meinungsverschiedenheiten bilden, ohne dass dies hinreichend aufgedeckt und berücksichtigt wird. Einige verstehen unter einer Moral, ganz so, wie es Soziologen, Historiker und Ethnologen tun, ein Ensemble sozialer Normen, die in einer Gesellschaft gelten und von ihren Mitgliedern ein bestimmtes Verhalten verlangen. Andere denken an etwas anderes. Für sie geht es bei einer Moral durchaus auch darum, dass man bestimmte Handlungen tun oder unterlassen muss, aber nicht, weil soziale Normen es verlangen, sondern weil diese Handlungen, normunabhängig, eine bestimmte Eigenschaft haben: Sie genügen, wie ein im Kopf durchgeführtes Testverfahren zeigt, einem vorausgesetzten Kriterium oder genügen ihm nicht, oder sie entsprechen einem vorab definierten moral point of view oder tun es nicht. Wieder andere nehmen an, bestimmte Handlungen hätten objektiv, das heißt unabhängig von uns, die Eigenschaft, moralisch getan oder unterlassen werden zu müssen. Die Moral sei eine objektive Ordnung, die unabhängig von sozialen Normen und unabhängig von subjektiven Kriterien existiert und uns verpflichtet, ihr gemäß zu handeln.

Diese verschiedenen Sichtweisen und die mit ihnen entstehenden assoziativen Kraftfelder kommen immerhin darin überein, dass eine Moral bedeutet,

*Kontakt: Peter Stemmer, Universität Konstanz, Fachbereich Philosophie, 78457 Konstanz; peter.stemmer@uni-konstanz.de 
dass man bestimmte Dinge tun oder unterlassen muss, und zwar, zumindest im Wesentlichen, um der anderen willen. Man muss ein gegebenes Versprechen halten, man darf einen anderen nicht töten, nicht verletzen, nicht demütigen, nicht gegen seinen Willen zu etwas zwingen, etc. All dies steht, so die Vorstellung, nicht im Belieben des Einzelnen, er muss es tun bzw. unterlassen, er ist, wie man auch sagt, dazu verpflichtet. Dieses Gefüge des Müssens ist vielleicht nicht das Ganze der Moral, aber doch ihr Kern.

Angesicht dieser Übereinstimmung und andererseits der untergründigen Differenzen im Verständnis muss die Frage, von welcher Art dieses Müssen ist und wodurch es in die Welt kommt, eine der ersten Fragen der Moralphilosophie sein. Umso überraschender ist es, dass sie nur selten ausdrücklich gestellt wird. Ein reflektiertes Verständnis der Moral muss diese Frage angehen: denn ohne eine Antwort wissen wir nicht, mit was für einem Phänomen wir es eigentlich zu tun haben. Wir leben mit etwas, und es ist uns wichtig, aber wir wissen nicht, was es ist.

Ich möchte im Folgenden einige Bemerkungen zu dieser Frage machen. Der Anlass ist das Erscheinen des Buches Moral und Sanktion. Eine Kontroverse über die Autorität moralischer Normen, herausgegeben von Eva Buddeberg und Achim Vesper, Frankfurt am Main 2013. Die Autoren der in diesem Band gesammelten Beiträge wenden sich fast unisono gegen eine Sanktionstheorie des moralischen Müssens, also gegen eine Theorie, die das moralische Müssen als durch Sanktionen konstituiert und damit als ein soziales Phänomen versteht. Da ich in verschiedenen Schriften eine solche Konzeption vertreten und verteidigt habe und meine Auffassungen deshalb deutlich kritisiert werden, möchte ich auf dieses Thema, nicht systematisch, aber mit einigen Überlegungen zurückkommen und etwas zu den Einwänden sagen, die mir die dringlichsten zu sein scheinen. ${ }^{1}$

\section{1}

Das Wichtigste ist zunächst, zwei äußerst einflussreiche Auffassungen zurückzuweisen. Zum einen den Objektivismus und zum anderen alle Varianten der Idee, das moralische Müssen sei ein kategorisches Müssen. Beide Auffassungen sind, so meine ich, fundamental falsch. Beide sprechen von Dingen, die es nicht gibt.

1 Weitere kritische Arbeiten, auf die ich hinweisen will, sind: Seebaß (2002); Engländer (2013); Freitag (2017). 
Die objektivistische Konzeption nimmt, wie gesagt, an, bestimmte Handlungen hätten an sich selbst, ohne einen subjektiven Anteil, die Eigenschaft, moralisch getan oder unterlassen werden $\mathrm{zu}$ müssen; wobei dieses Müssen offenkundig kein naturgesetzliches Müssen ist, auch kein logisches, sondern ein normatives. Das heißt, es ist mit einem Handlungsdruck verbunden. Wer etwas moralisch tun muss, steht unter einem Druck, so zu handeln. Kant hat dieses Merkmal zum Ausdruck gebracht, indem er von „Imperativen“ sprach. Die These des Objektivismus ist also, dass bestimmte Handlungen die Eigenschaft eines solchen normativen Gemusstseins an sich selbst haben.

Eine Handlung kann von der Art sein, dass sie getan werden muss dafür, dass eine bestimmte Konsequenz eintritt; und diese Notwendigkeit kann ohne Zweifel unabhängig von uns bestehen. Aber ein solches Müssen ist nur ein naturgesetzliches oder sonstiges - Müssen der notwendigen Bedingung, jedoch kein normatives Müssen. Das normative Müssen ist mehr, es ist, wie gesagt, mit einem Handlungsdruck verbunden. Die einschlägigen Handlungen müssen objektiv, unabhängig von uns - diese Eigenschaft des normativen Gemusstseins oder, anders gesagt, des Imperativischen haben. Mackie hat von einer „objective prescriptivity “ gesprochen. ${ }^{2}$ Dabei kann es sich nur um eine überempirische Eigenschaft handeln, eine überempirische Eigenschaft, die etwas Empirischem, eben den Handlungen, inhäriert. Noch niemand hat erläutern können, von welcher Art eine solche Eigenschaft ist. Wir kennen solche Eigenschaften nicht und haben nicht die leiseste Vorstellung von ihrer Natur. An diesem elementaren ontologischen Problem scheitert, so meine ich, der Objektivismus. Tatsächlich präsentiert er uns nur eine Erfindung, etwas, was es in Wirklichkeit nicht gibt.

Es ist gewiss so, dass einem die Moral zunächst als etwas objektiv Vorgegebenes erscheint. Man erlebt sie nicht als etwas, zu dessen Wirklichkeit und Bedeutung man selbst in irgendeiner Weise beiträgt. Man erlebt sie als eine Ordnung des Müssens und Nicht-Dürfens, die einfach „da“ ist und der man zu entsprechen hat. Umso wichtiger ist es, sich klarzumachen, dass diese Objektivität keine ontologische Objektivität ist, dass die Tatsache, dass man eine bestimmte Handlung moralischerweise unterlassen muss, also keineswegs subjektunabhängig ist, vielmehr eine - im Einzelnen zu erläuternde - subjektive Ontologie hat.

Die objektivistische Konzeption versteht das moralische Müssen notwendigerweise als kategorisch, also als wollensirrelativ. Aber auch jenseits des Objektivismus, bei Kant und seinen Nachfolgern, wird das moralische Müssen, jetzt in einer subjektivistischen Variante, als kategorisch verstanden. Doch diese Idee, gleichgültig in welcher Form, operiert ebenfalls mit etwas, was es nicht gibt.

2 Mackie (1977), 35, 42, 45, 47 u. 48. 
Auch hier hat noch niemand zeigen können, dass so etwas existiert. Auch hier handelt es sich um eine Chimäre, von der die Philosophie, wie es scheint, nicht loskommt.

Ein normatives Müssen und der mit ihm verbundene Handlungsdruck ist dann gegeben, wenn gilt: Wenn man anders handelt, anders als „gemusst“, passiert etwas Negatives. Oder: Nur, wenn man wie „gemusst“ handelt, passiert etwas Positives. Negativ ist etwas aber dadurch, dass man es nicht will, positiv dadurch, dass man es will. Wenn ich den Wunsch habe, einen Marathon zu laufen, muss ich dafür trainieren. Ich stehe dann unter einem Druck, zu trainieren. Dieses Müssen ist ein normatives Müssen. Und es ist umso stärker, je stärker mein Wollen ist. Verhalte ich mich anders als „gemusst“, ist die unweigerliche negative Konsequenz, dass ich nicht erreiche, was ich will. Und genau dies, die Unausweichlichkeit der negativen Konsequenz, erzeugt den für ein normatives Müssen typischen Handlungsdruck. Nimmt man das Wollen weg - ich habe noch nie daran gedacht, einen Marathon laufen zu wollen, das liegt mir völlig fern -, ist es zwar immer noch so, dass ich trainieren muss dafür, dass ich den Marathon laufen kann, das ist einfach eine biologische Tatsache, aber dieses Müssen ist dann kein normatives, es ist ein bloßes Müssen der notwendigen Bedingung, das mich nicht berührt. Es geht mich nichts an, es hat keinen imperativischen Charakter.

Es ist wichtig, hier einen zusätzlichen Aspekt einzuführen. Wenn ich einen Marathon laufen will und dafür trainieren muss, stehe ich nicht nur unter einem Druck, zu trainieren, ich habe auch einen Grund, dies zu tun, es spricht etwas dafür. Wo ein normatives Müssen, so kann man sagen, da ein Handlungsgrund. Und warum spricht etwas dafür, zu trainieren? Was konstituiert diesen Grund? Die Tatsache, dass ich, wenn ich anders handle, etwas, was ich will, nicht erreiche. Das Entscheidende ist also erneut die Struktur: wenn nicht, dann etwas Negatives. Oder: nur wenn, dann etwas Positives. Ohne einen Wollensbezug, der, wie gesagt, in der Rede von „positiv“ und „negativ“ impliziert ist, lässt sich gar nicht explizieren, was es heißt, für etwas zu sprechen und ein Grund zu sein. ${ }^{3}$

Wer nun behauptet, es könne ein kategorisches normatives Müssen geben, muss zeigen, wie es möglich ist, dass ein solches Müssen ohne Wollensbezug mit einem Handlungsdruck verbunden sein kann, wie es also einen imperativischen Charakter haben kann. Wenn einem gesagt wird, dass man etwas tun muss, kann man immer zurückfragen: „Und was passiert, wenn ich es nicht tue, wenn ich anders handele?“ Wenn die Antwort lautet: „Es passiert nichts“, worin besteht

3 Vgl. hierzu Stemmer (2013d), bes. 140-141. 
dann das normative Müssen? Wodurch kommt es dann zu einem Druck, sich wie „gemusst“ $z$ u verhalten? Diese Frage bleibt, so scheint mir, ohne Antwort. ${ }^{4}$

Wer die Möglichkeit eines kategorischen normativen Müssens behauptet, muss auch zeigen, wie ein solches Müssen einen Grund darstellen kann, einen Grund, der dafür spricht, sich wie „gemusst“ zu verhalten. Worin besteht dieser Grund, wenn nicht darin, dass, wenn man anders handelt, etwas Negatives passiert? Wodurch hat man, obwohl dies nicht passiert, gleichwohl einen Grund, sich so zu verhalten? Auch diese Frage bleibt ohne Antwort.

Vielen liegt an dieser Stelle auf der Zunge, dass man einen Grund hat, so zu handeln, weil man einsieht, dass die Handlung vernünftig ist. Aber damit kommt es nur zu einer irreführenden Verdoppelung der Rede vom Grund. Denn dass man einen Grund hat, eine Handlung zu tun, weil man einsieht, dass sie vernünftig ist, heißt, dass man einen Grund hat, sie zu tun, weil man einsieht, dass etwas für sie spricht, man also einen Grund hat, sie zu tun. In Kurzform: Man hat einen Grund, weil man einen Grund hat. Diese Strategie führt offensichtlich zu nichts und vernebelt die Dinge nur. Die entscheidende Frage ist nach wie vor, wodurch man, obwohl, wenn man anders handelt, nichts Negatives passiert, einen Grund hat. Und diese Frage bleibt, wie gesagt, unbeantwortet.

Es kommt noch eine weitere Überlegung hinzu. Ein normatives Müssen zeigt die irritierende Eigenheit, dass man einerseits muss, andererseits aber doch anders kann. Man muss sein Versprechen halten, und doch kann man es, wie jeder weiß, brechen. Etwas zu müssen, bedeutet, dass man nicht anders kann, dass es keine andere Möglichkeit gibt, dennoch kann man anders. Ich habe diese Merkwürdigkeit „das Paradox des normativen Müssens“ genannt. Es hat viele Philosophen verwirrt, und sie haben, um sich daraus $\mathrm{zu}$ befreien, gewöhnlich einen Weg eingeschlagen, der, ich komme darauf noch, in einer Sackgasse endet. Tatsächlich löst sich das Paradox auf, wenn man sich klarmacht, dass ein normatives Müssen immer wollensrelativ ist. Wenn ich jetzt aufbrechen muss, um den Zug um 14.00 Uhr nach Urnäsch zu erreichen, besteht tatsächlich keine andere Möglichkeit. Ich muss, wenn ich den Zug erreichen will, jetzt aufbrechen. Das ist ein echtes Müssen. Dennoch kann ich noch bleiben. Das steht in meiner Macht. Allerdings erreiche ich dann, das ist die unausweichliche Konsequenz, den Zug nicht. Ich kann also noch bleiben, aber ich kann nicht bleiben und doch den Zug erreichen. Das ist unmöglich. Dies zeigt, dass das Müssen ein echtes Müssen ist, aber relativ ist auf ein Wofür. Die Optionslosigkeit, die das Müssen bedeutet, betrifft also nicht die gemusste Handlung - das Jetzt-Aufbrechen - an und für sich. Die Optionslosigkeit besteht darin, dass es ausgeschlossen ist, anders als

4 Vgl. hierzu eingehender ders. (2000), $59 \mathrm{ff}$. 
„gemusst“ zu handeln und doch ein bestimmtes Ziel zu erreichen. Die Lösung des Paradoxes liegt also offenkundig darin, dass das Müssen bedingt oder relativ ist. Es ist ein Müssen dafür, dass. Man muss etwas tun dafür, dass etwas geschieht oder nicht geschieht. Wer annimmt, ein normatives Müssen könne kategorisch sein, muss wiederum zeigen, wie es möglich ist, dass ein Müssen, das nicht auf ein Wofür bezogen ist, dennoch damit vereinbar ist, dass man anders kann. Das ist, so denke ich, ganz ausgeschlossen. Der einzige Ausweg, der sich bietet, ist, zu sagen, die Rede vom Müssen sei nicht ernst gemeint, es sei nur eine façon de parler, die man, wenn nachgefragt werde, schnell zurücknehme.

Auf dieser Linie kann man gegen das letzte Argument einwenden, es setze voraus, dass ich vom Müssen und nicht vom Sollen spreche. Wenn man vom Sollen ausgehe, entstehe diese Schwierigkeit erst gar nicht. Denn beim Sollen sei es unproblematisch, dass man anders handeln kann. Tatsächlich ist genau dies der Weg, den die meisten Philosophen angesichts des für das normative Müssen typischen Paradoxes einschlagen. Wir sprechen, so sagen sie, nicht vom Müssen, nicht vom Nicht-anders-Können, sondern von einem Sollen. Und dann kommt es nicht zu diesem Problem. Doch dieser Zug endet, wie schon gesagt, in einer Sackgasse. $^{5}$

Man kann hier zunächst festhalten, dass Kant sehr deutlich sowohl bei den hypothetischen wie auch bei den kategorischen Imperativen von einer praktischen Notwendigkeit spricht. ${ }^{6}$ Es geht also, in und außerhalb der Moral, um ein Müssen. Kant spricht zwar auch von einem Sollen, aber er meint damit ein - spezielles - Müssen. „Das Sollen“, so heißt es in der Kritik der reinen Vernunft, „drückt eine Art von Notwendigkeit [...] aus, die in der ganzen Natur nicht vorkommt. “7 Das „Sollen“ bezeichnet also ein Müssen, allerdings nicht das naturgesetzliche Müssen, sondern das normative Müssen, und hier vom „Sollen“ zu sprechen, hat gerade die Funktion, den normativen (oder imperativischen) Charakter dieses Müssens hervorzuheben, also das, was ich den Handlungsdruck genannt habe. Kant interessiert sich nicht für die sprachlichen Details, aber seine Formulierungen zeigen deutlich, dass er an ein Müssen denkt.

Wenn man das Sollen hingegen, anders als Kant, beim Wort nimmt und darunter etwas anderes als ein normatives Müssen versteht, was ist dann gemeint? Dann spricht man von etwas ganz anderem. Dass man etwas tun soll, bedeutet, dass jemand will, dass man so handelt. Wenn die Zeitung meldet: Der Bundes-

5 Vgl. hierzu ders. (2008), 284-295.

6 Kant (1911), 414-416 u. 418.

7 Ders. (1781/1787), A 547, Hervorh. im Orig. So auch schon wesentlich früher, 1764: „Nun drückt jedes Sollen eine Notwendigkeit der Handlung aus“, ders. (1912), 298. 
tag soll die Asylbeschleunigungsgesetze noch vor der Sommerpause verabschieden, bedeutet das, dass jemand dies will - vielleicht der Bundestagspräsident, die CDU-Fraktion oder der Bundesinnenminister. Wenn es hingegen heißt: Der Bundestag muss die Asylbeschleunigungsgesetze noch vor der Sommerpause verabschieden, liegt ein ganz anderer Sachverhalt vor. Dann besteht die Notwendigkeit, das Gesetzespaket noch vor der Pause zu beschließen, etwa weil sonst die untragbaren Zustände in den Asylunterkünften fortbestehen oder weil die Gefahr besteht, dass sich nach den Herbstwahlen die Mehrheitsverhältnisse verändert haben, oder weil sonst etwas Negatives geschehen wird. Das Sollen rekurriert auf das Wollen eines anderen. Wobei im Soll-Satz ungesagt bleibt, wer das Subjekt des Wollens ist. Soll-Sätze sind in diesem Sinne rekursiv und anonym. Den Rekurs auf einen anonym bleibenden anderen finden wir auch in einem anderen, aber parallelen Gebrauch des Wortes „sollen“. Wenn jemand sagt: Bei der Insolvenz der Firma Moser soll nicht alles mit rechten Dingen zugegangen sein, bedeutet das: Jemand behauptet, oder es wird behauptet, dass nicht alles mit rechten Dingen zugegangen ist. Hier verweist das Sollen nicht auf das Wollen, sondern auf die Behauptung eines anderen. ${ }^{8}$

Es hängt nun viel daran, zu sehen, dass ein an mich adressiertes Wollen eines anderen, also ein Sollen mich nicht zu irgendetwas nötigt. Wenn ein anderer will, dass ich etwas tue, muss mich das nicht kümmern. Viele Leute wollen etwas von mir. Ein fremdes Wollen bedeutet nicht, dass ich unter Druck bin, entsprechend $\mathrm{zu}$ handeln. Ich kann immer fragen, warum mich das Wollen eines anderen etwas angehen sollte. Es ist sein Wollen, nicht mein Wollen. Anders gewendet kann man sagen: Ein fremdes Wollen ist, für sich genommen, kein Grund, so zu handeln, wie es der andere will. Das ändert sich nur, wenn zusätzliche Elemente hinzutreten, zum Beispiel, wenn ich dem anderen entgegenkommen will, oder wenn ich ihm etwas schuldig bin, oder wenn er eine besondere Autorität besitzt. Das Wollen des anderen ist, so zeigt sich, nicht normativ. Wenn wir das Sollen beim Wort nehmen und, anders als Kant, darunter nicht ein Müssen verstehen, sprechen wir von etwas, das die Eigenschaft des Normativen gar nicht hat. Damit ist bereits gesagt, dass auch die moralische Normativität durch diese Rede vom Sollen nicht erfasst wird. Die Moral setzt uns unter Druck, in bestimmter Weise zu handeln, ihre Gebote haben einen imperativischen Charakter. Ein Sollen, sprich: das Wollen eines anderen, hat dies aber gerade nicht. Es ist etwas ganz anderes.

Wenn man meint, das Müssen sei zu stark, weil man ja anders kann, das Sollen aber zu schwach ist, was bleibt dann, um die Normativität zu verstehen? Was gibt es dazwischen? Diese Frage muss man beantworten, wenn man ange-

8 Vgl. zur Analyse des „Sollens“ im Einzelnen Stemmer (2000), 39-48. 
sichts des Paradoxes des normativen Müssens die Rede vom moralischen Müssen fallen lassen will. Tatsächlich gerät man mit dieser Strategie in ein Niemandsland, in dem sich nichts findet, was einem zu einer Antwort verhelfen könnte. Man könnte vielleicht an ein „man sollte“ denken: Man sollte so-und-so handeln. Es ist jedoch, besonders im Blick auf die Moral, ohne Weiteres klar, dass es nicht darum geht, einer Empfehlung oder einem Rat zu folgen.

Man könnte auch, und viele gehen wohl in diese Richtung, daran denken, dass es zwischen dem Sollen im Wortsinne und dem normativen Müssen so etwas wie Forderungen gibt und dass mit der Rede vom Sollen gewöhnlich genau hieran gedacht sei. Man spricht ja oft von moralischen Forderungen, und von jemandem etwas zu fordern, sei offensichtlich mehr als, von ihm nur etwas zu wollen. Aber was ist dieses Mehr? Man darf nicht glauben, dass jemand schon dadurch etwas fordert, dass er sagt: „Ich fordere von dir, das-und-das zu tun.“ Etwas zu fordern, setzt eine bestimmte Situation voraus. Der, der fordert, muss dem anderen gegenüber über bestimmte Machtmittel verfügen und für den Fall des Anders-Handelns glaubhaft mit negativen Konsequenzen drohen können. Ohne dieses Element haben wir es mit einem bloßen Wollen zu tun, das sich als Forderung ausgibt und eine Stärke vortäuscht, die es tatsächlich nicht hat. Wenn eine Forderung aber so zu verstehen ist, wie jetzt wenigstens angedeutet ${ }^{9}$, dann kommt in ihr ein wollensrelatives normatives Müssen zum Ausdruck: Man muss sich so verhalten, wenn man nicht will, dass etwas Negatives passiert. Wir finden also erneut nicht, was gesucht wird: eine „Sollen“ betitelte schwächere Variante des Normativen neben dem normativen Müssen.

Die philosophische Rede vom Sollen hat sich, soweit ich sehe, erst im Laufe des 19. Jahrhunderts in der deutschen nachkantischen Philosophie etabliert, vermutlich aus dem Bestreben, das normative Müssen durch einen eigenen Terminus vom naturgesetzlichen Müssen abzuheben. ${ }^{10}$ Genauso, wie man begann, statt von praktischen Gesetzen von Normen zu sprechen, vermutlich ebenfalls um praktische Gesetze und Naturgesetze zu unterscheiden. Sofern mit dem Sollen etwas anderes als ein Müssen gemeint war, hat man, so scheint mir, nie zu sagen vermocht, woran man denkt. Und sofern man mit dem Sollen doch ein Müssen meinte, eben das spezifisch normative Müssen, hat man die Natur dieses Müssens auch nie klären können. Stattdessen hat man das Sollen mystifiziert und zu einer eigenen, höheren Wirklichkeit jenseits des „Seins“ gemacht, aus der es dann auf uns herabkommt. Diese Tradition des Neukantianismus hat bis

9 Vgl. genauer ebd. (2000), 50-53; ders. (2008), 108, 167-170, 273.

10 Kant spricht bereits davon, dass „das Sollen oder der Imperativ“ „das praktische Gesetz vom Naturgesetz unterscheidet“; vgl. Kant (1923), 13. 
heute erheblichen Einfluss auf die Theoriebildung in der Moralphilosophie und der praktischen Philosophie in Deutschland.

Wenn man sich klarmacht, dass es kein kategorisches normatives Müssen gibt, dass jedes normative Müssen wollensrelativ ist und dass sich das Paradox des normativen Müssens deshalb leicht auflöst, entfällt der Impuls, statt vom Müssen von einem vermeintlich schwächeren Sollen zu sprechen.

Warum aber ist die Vorstellung, das moralische Müssen sei kategorisch, für viele so selbstverständlich? Das hat verschiedene Gründe. Ich nenne hier nur zwei. Zunächst nehmen wir alle an, dass man sein Versprechen halten muss, unabhängig davon, ob man es halten will oder nicht. Dass das moralische Müssen von diesem, auf das „Gemusste“ selbst gerichteten Wollen unabhängig ist, wird allerdings von keiner Theorie des moralischen Müssens in Frage gestellt. Es wäre ein Missverständnis, anzunehmen, dass dies von einer Konzeption, die das moralische Müssen als wollensrelativ versteht, bestritten würde. Denn auch hypothetische Imperative sind offensichtlich von diesem, auf das „Gemusste“ selbst gerichteten Wollen unabhängig. Wenn ich den Zug um 14.00 Uhr erreichen will, muss ich jetzt aufbrechen, gleichgültig, ob mir das gefällt oder nicht. Wenn sich diese Selbstverständlichkeit, dass man muss, unabhängig davon, ob man will, dann aber zu der Intuition steigert, das moralische Müssen sei von jeglichem Wollen unabhängig, hat das seinen Grund wenigstens zum Teil darin, dass wir als Kinder lernen, dass man bestimmte Handlungen nicht tun darf und andere tun muss. Dies wird uns vermittelt als etwas, das einfach so ist, als so etwas wie ein natürliches Gesetz. Man macht zwar irgendwann die Erfahrung, dass es möglich ist, sich auch gegen das moralisch „Gemusste“ zu verhalten. Wenn man dies tut, stellt sich jedoch das unangenehme Gefühl ein, damit gegen eine wie immer geartete objektive Ordnung zu verstoßen, die ganz unabhängig von unserem Wollen existiert. Wir sind intuitiv Objektivisten, und der Objektivismus impliziert die Vorstellung des Kategorischen.

Der zweite Grund: Bei Kant wird die völlige Dissoziation von Moral und Wollen gestützt durch die für seine Philosophie grundlegende Abwertung des Wollens. Das Wollen, die natürlichen Antriebe und Neigungen sind, ganz in der platonischen Tradition, etwas Fremdes in uns, etwas bloß Naturhaftes, etwas Niedriges, etwas, was uns, wenn wir uns davon in unserem Handeln bestimmen lassen, versklavt und in die Heteronomie stürzt. Wir würden nach fremden Gesetzen leben, die nicht wir uns gegeben haben, die vielmehr die Natur uns vorgibt und denen wir bloß unterworfen wären. ${ }^{11}$ Nur dadurch, dass wir unsere Hand-

11 Ich habe diese Abwertung des Wollens und die damit einhergehende Theoriebildung kritisiert in Stemmer (2016), § 8. 
lungen durch kategorische, gänzlich wollensunabhängige Imperative bestimmen können, erheben wir uns über die Natur und ihre Gesetze. Man kann sich zwar auch außerhalb der Moral Maximen für sein Handeln geben, das ist ohne Zweifel ein Stück Selbstbestimmung, aber diese Maximen dienen natürlichen Antrieben und Neigungen. Sie haben also nur eine instrumentelle, auf diese Antriebe bezogene Funktion. Deshalb entsteht mit ihnen zwar eine komplexere Form der Handlungssteuerung, aber nichts grundsätzlich anderes. Alles Handeln bleibt, mehr oder weniger vermittelt, letztlich durch die naturhaften Wünsche bestimmt. Der wirkliche Überstieg auf das Niveau des menschlichen Lebens und das eines Vernunftwesens ist nur möglich, wenn es kategorische, von allem Wollen unabhängige Imperative gibt und die Menschen ihr Verhalten durch diese Imperative zu bestimmen vermögen. Diese Freiheit von der Herrschaft oder, wie Kant auch sagt, dem „Gängelband“ der Natur erreichen die Menschen nur in der Moral. Denn nur in der Moral gibt es kategorische Imperative. Nur hier arbeiten sie sich aus der Determination durch die Naturgesetze heraus und gewinnen wirkliche Selbstbestimmung.

Wenn man sich diesen Kontext vor Augen führt, zeigt sich deutlich, dass Kants Annahme kategorischer Imperative nicht nur das entscheidende Element seiner Moralphilosophie darstellt, sondern eine weit darüber hinausreichende Bedeutung hat. Sie ist das zentrale Element in der Selbstbehauptung des Menschen. Nur weil wir fähig sind, unser Verhalten an kategorischen Imperativen auszurichten, sind wir nicht nur besonders komplizierte Tiere, sondern grundsätzlich andere Wesen. Diese Motive, die sich mit dem Theoriestück der kategorischen Imperative weit über die Moral hinaus verbinden, sind auch für viele heutige Philosophen attraktiv. Wenn man die Kategorizität des Moralischen opfern müsste, so die Angst, würde man nicht nur die Moral anders verstehen müssen, auch das Selbstverständnis des Menschen müsste sich einschneidend ändern, und zwar in eine Richtung, in die man auf keinen Fall will. Die Vorstellung des kategorischen Müssens wird also nicht nur von Intuitionen getragen, die aus der Sozialisation kommen, sondern aus sehr viel breiteren Interessen, die auf ein bestimmtes Programm der Selbstbehauptung des Menschen zielen.

All dies ändert freilich nichts daran, dass es ein kategorisches, wollensirrelatives normatives Müssen nicht gibt und dass es weder Kant (der sich der Schwierigkeiten sehr bewusst war) noch seinen Nachfolgern gelungen ist, diese Idee plausibel zu machen. Es handelt sich um ein philosophisches Konstrukt, geboren aus dem Wunsch, die Menschen wenigstens zum Teil aus der Determination durch die Natur herauszulösen und sie auf diese Weise zu einem höheren Wesen zu machen. Wenn wir eine Auffassung des moralischen Müssens entwickeln wollen, die sich von Illusionen freimacht, müssen wir die Idee eines kategorischen moralischen Müssens fallen lassen. 


\section{2}

Wenn wir außerdem die Vorstellung eines objektiven normativen Gemusstseins von Handlungen aufgeben, bleibt nur, auch das moralische Müssen als wollensrelativ zu verstehen. Seine Existenz hängt von einem Wollen ab, und zwar von einem Wollen dessen, der muss. Das Moralische zu tun, ist also notwendig dafür, etwas Gewolltes zu erreichen oder etwas Nicht-Gewolltes zu vermeiden. Die moralische Normativität ist damit durch genau die beiden Elemente konstituiert, die für alle Normativität definitiv sind: (i) ein Müssen der notwendigen Bedingung und (ii) ein Wollen. ${ }^{12}$ Man will $y$, und wenn das Tun von $x$ notwendig ist dafür, dass $y$ geschieht, dann muss man $x$ tun. Dann hat man einen Grund, $x$ zu tun, es spricht etwas dafür; und dann hat man auch ein Motiv, $x$ zu tun, und man steht unter einem Druck, $x$ zu tun.

Wenn der Wunsch, auf den das Müssen relativ ist, von der Art ist, dass es gar nicht möglich ist, ihn nicht zu haben, wenn wir ihn aufgrund unserer biologischen Konstitution notwendigerweise haben, es sich also, wie Harry Frankfurt gesagt hat, um eine volitionale Notwendigkeit handelt, dass wir diesen Wunsch haben, dann stehen wir unausweichlich unter dem entsprechenden Müssen. Wir können uns ihm nicht entziehen, indem wir das vorausgesetzte Wollen fallen lassen. Die Menschen haben eine ganze Reihe solcher Wünsche. Und wir werden sehen, dass das moralische Müssen gerade auf ein solches Müssen bezogen ist. Oft wird gesagt, es könne nicht sein, dass das moralische Müssen von Wünschen abhängig sei, diese seien „kontingent“ oder „,arbiträr“. Aber das übersieht, dass wir basale Wünsche haben, von denen wir uns nicht losmachen können.

Innerhalb des jetzt skizzierten Rahmens gibt es verschiedene Möglichkeiten, das moralische Müssen zu verstehen. Man kann zwei Theorietypen unterscheiden. Auf der einen Seite stehen Theorien, die annehmen, die Umstände, in denen die Menschen leben, und ihre Wünsche seien ohne weiteres Arrangement so, dass moralisches Verhalten eine notwendige Bedingung für etwas ist, was sie wollen. Es besteht, so die Annahme, eine Harmonie zwischen der Moral und dem, was die Menschen wollen. Deshalb hat jeder, so wie die Welt ist, Gründe, sich moralisch zu verhalten. Eine klassische Ausformung dieser Konzeption ist der antike Eudaimonismus. Hier muss man sich moralisch verhalten dafür, glücklich zu werden, etwas, was jeder will. Wenn man nur die richtige Vorstellung davon hat, worin das menschliche Glück besteht, kann man erkennen, dass die Moral ein notwendiges Ingrediens (oder mehr) eines glücklichen Lebens ist. Das ist einfach so, man muss es nur aufdecken.

12 Vgl. hierzu im Einzelnen Stemmer (2008), § 4. 
Eine andere Spielart dieser Auffassung entwickeln Rational-Choice-Theorien der Moral. Bestimmte Formen des moralischen Kontraktualismus sind hierfür ein Beispiel. Die Menschen können, so die Idee, zu erheblichen Verbesserungen ihres Lebens kommen, wenn sie miteinander kooperieren. Um dahin zu kommen, muss sich allerdings jeder, vorausgesetzt, die anderen tun das auch, bestimmten Handlungsbeschränkungen unterwerfen. Man muss eigene Interessen zugunsten anderer zurückstellen, sprich: sich moralisch verhalten. Auch hier ist es so, dass das moralische Verhalten ein Segment dessen ist, was man tun muss, um bestimmte Ziele zu erreichen, allerdings, darin liegt eine Differenz zum Eudaimonismus, nur unter der Bedingung, dass die anderen sich auch so verhalten. Wenn man sich die Baugesetze kooperativen Verhaltens vor Augen führt, erkennt man also, dass man sich moralisch verhalten und entsprechende Dispositionen ausbilden muss. Auch hier besteht aufs Schönste eine Harmonie von Moral und dem Interesse am eigenen Wohl. Man muss sie nur aufdecken.

Auf der anderen Seite nimmt man an, dass es diese prästabilierte Harmonie von Moral und eigenen Interessen nicht gibt, oder nicht durchgängig gibt, oder oft nicht hinreichend klar ist, ob sie besteht. Wenn man, anders als Aristoteles, nicht daran glaubt, dass, was das menschliche Glück ausmacht, durch so etwas wie eine kosmische Ordnung objektiv fixiert ist, vielmehr meint, die Menschen könnten, ohne dass daran etwas falsch ist, verschiedene Vorstellungen vom Glück haben, scheint es sehr fraglich zu sein, ob für alle gilt, dass sie sich moralisch verhalten müssen, um das, was sie als ihr Glück anstreben, zu erreichen. Thrasymachos hat im ersten Buch der Politeia eindringlich dafür argumentiert, dass es, wenn man „Glück“ so versteht, wie er es für naheliegend hält (und nicht so, wie Platon es dann entwickelt), keineswegs so ist, dass man sich moralisch verhalten muss, um glücklich zu leben. Es sei genau umgekehrt, moralisches Verhalten sei nicht glückszuträglich, sondern glücksabträglich.

Und auch wenn man nicht das Glücksstreben, sondern ein anderes Wollen zugrunde legt, bestehe, so diese Konzeption, die Harmonie von Moral und eigenem Wollen nicht in der unterstellten Art. Sie bestehe in vielen Fällen nicht, und in anderen Fällen könne man nicht wissen, ob sie besteht, weil man nicht wirklich weiß, wie sich die anderen verhalten werden. Oder es gibt zwar Gründe dieser Art für die Moral, aber sie sind nicht stark genug. Wie immer die Diagnose der Defizite genau ausfällt, es ist in jedem Fall, um sicher zu gehen, nötig, künstliche Gründe für moralisches Verhalten zu schaffen, also das moralische Handeln künstlich auf die Linie des Wollens zu manövrieren. Und zwar auf die Linie eines Wollens, das jeder hat und das sehr stark ist, so dass möglichst jeder möglichst in allen Situationen einen möglichst starken Grund hat, sich moralisch zu verhalten.

Wie schafft man ein solches künstliches Müssen? Dadurch, dass man die Handlungen, die nicht getan werden sollen, künstlich mit einer negativen Konsequenz, 
also mit einer Sanktion verknüpft. Geschieht dies, muss man sich moralisch verhalten, um dieser negativen Konsequenz zu entgehen. Die Sanktion ist umso effektiver, je elementarer und stärker der Wunsch ist, an den sie andockt. Der Wunsch, der hier am besten geeignet ist, ist der Wunsch nach Anerkennung und Akzeptanz durch die anderen. Dies ist ein basaler Wunsch der Menschen, jeder hat ihn, weit unterhalb jeder speziellen Glückskonzeption. Er ist äußerst stark, und es ist nicht möglich, sich von ihm loszumachen. Die Sanktion muss also darin bestehen, Anerkennung zu entziehen. Die anderen reagieren negativ, ablehnend, man verliert in ihren Augen Anerkennung und Wertschätzung, und sie zeigen einem das. Und selbst wenn sie es nicht zeigen, einen vielleicht nur in Gedanken herabstufen, ist dies schon etwas, was uns empfindlich stört, etwas, was wir gerne vermeiden würden.

Dieser zweite Theorietyp versteht die Moral als ein System künstlicher Gründe. Die Gründe zur Moral sind folglich Artefakte, Ergebnis menschlichen Arrangements. Und die Funktion der Moral ist es, auf diese Weise in die Überlegung ein Gewicht oder ein zusätzliches Gewicht zugunsten des moralischen Verhaltens einzubringen und die Menschen unter Druck zu setzen, sich entsprechend zu verhalten. Wenn eine solche Sanktionspraxis in einer Gesellschaft entsteht, spricht man davon, dass es in ihr informelle Normen gibt, die es verbieten, die einschlägigen Handlungen $\mathrm{zu}$ tun oder zu unterlassen. Die Moral wird hier also als ein Ensemble informeller sozialer Normen verstanden.

\section{3}

Diese zweite Auffassung versteht das moralische Müssen, wie sich zeigt, als sanktionskonstituiert. Es mag unterhalb des künstlich geschaffenen Müssens bereits ein anderes Müssen mit demselben Gegenstand geben, etwa eines der rationalen Kooperation, aber das spezifisch moralische Müssen, das, mit dem die Moral erst entsteht, ist hiernach das durch die Sanktion künstlich geschaffene.

Mit dieser Auffassung verbindet sich ein soziales oder, wie man auch sagen kann, institutionelles Verständnis der Moral. Die Moral ist eine soziale Errungenschaft, eine Institution, zu der es kommt, weil man mit ihr sehr viel besser leben kann als ohne sie. In allen Gesellschaften sind solche informellen, mit sozialem Druck operierenden Normensysteme entstanden. Die fundamentale Idee der Moral, so sagt Peter F. Strawson, ist die „of a socially sanctioned demand made on an individual in virtue merely of the membership of the society in question“. ${ }^{13}$

13 Strawson (1974), 32. 
Dieses soziale Verständnis der Moral unterscheidet sich von vorsozialen Konzeptionen. Im Eudaimonismus muss man die Handlungen tun, die glückszuträglich sind, und dazu gehören, so will die Theorie zeigen, Handlungen, die anderen zugutekommen. Das Müssen ist hier ein „einfaches“, das heißt, nicht durch Normen künstlich geschaffenes hypothetisches Müssen. Genauso in den angesprochenen Rational-Choice-Theorien der Moral. Man muss, um ein hochstufiges Ziel zu erreichen, auch die Verfolgung eigener Interessen beschränken und sich zugunsten anderer verhalten. In diesen letzten Theorien ist eine gewisse soziale Komponente im Spiel, weil man dies nur muss, wenn die anderen sich genauso verhalten. Aber das ist bloß ein komplizierendes Element. Es ändert an der Natur des Müssens nichts. Das Müssen bleibt ein „einfaches“ hypothetisches Müssen, es ist nicht sozial, durch Sanktionen und informelle Normen konstituiert. In diesen Konzeptionen spielen offenkundig keine Gründe eine Rolle, die eigens zu dem Zweck geschaffen werden, Moral und eigenes Interesse auf eine Linie zu bringen.

Vorsozial sind auch Konzeptionen, nach denen man mögliche Handlungen an einem vorab definierten moral point of view oder einem vorab fixierten Moralkriterium misst. Man tut hier etwas im Kopf, man prüft im Kopf, ob eine Handlung einem Kriterium entspricht, und am Ende weiß man, was man tun und lassen muss. Die Moral besteht folglich darin, dass man über ein solches Kriterium verfügt, seine Handlungen daran prüft und entsprechend handelt.

In Konzeptionen dieser letzten Art bleibt, wie es scheint, unklar, wie das moralische Müssen zu verstehen ist. Warum muss man die Handlungen unterlassen, die dem vorausgesetzten Kriterium nicht entsprechen? Häufig hat man die Idee, dass man sich so verhalten muss, weil man moralisch handeln will. Wenn man das will, darf man die Handlungen, die dem Kriterium nicht entsprechen, offensichtlich nicht tun. Diese Antwort ist aber aus zwei Gründen (oder einem Grund mit zwei Seiten) nicht überzeugend. Zum einen ist das Müssen, das hier ins Spiel gebracht wird, nur so etwas wie ein Müssen der Subsumtion: Eine bestimmte Handlung erweist sich als unmoralisch, also muss man sie unterlassen, wenn man sich moralisch verhalten will. Es ist, so meine ich, sehr klar, dass dieses Müssen nicht das moralische Müssen sein kann. Außerdem ist das moralische Müssen nicht von dem Wunsch, moralisch zu sein, abhängig. Es bleibt also offen, wie in Konzeptionen dieser Art die moralische Normativität zu verstehen ist. 


\section{4}

Das moralische Müssen sozial zu verstehen, also als durch Sanktionen konstituiert, zieht eine wichtige Unterscheidung nach sich. Wenn eine Gesellschaft mit Hilfe von Sanktionen informelle Verhaltensnormen schafft, kann man in Bezug auf jede dieser Normen fragen, ob sie das Richtige sanktioniert, ob das, was sie verbietet, verboten sein sollte, und das, was sie gebietet, geboten. Aber selbst wenn jemand meint, eine Norm sei in diesem Sinne nicht in Ordnung, und es tatsächlich auch so ist, gibt es, solange die Sanktionspraxis fortbesteht, diese Norm und das mit ihr gegebene Müssen, und damit einen Grund, sich normkonform zu verhalten. Denn wenn man es nicht tut, wird man sanktioniert. Man muss folglich zwei Fragen deutlich auseinanderhalten: die Frage, wodurch eine Norm und das normgenerierte Müssen existiert, also die Frage der Existenz, und die Frage der Richtigkeit, also die Frage, ob die Norm richtig ist, ob sie das Richtige sanktioniert. Existenz und Richtigkeit sind zwei unterschiedliche Dinge. Und die Falschheit einer Norm nimmt nichts von ihrer Existenz. Auch eine schlechte oder falsche Norm ist noch eine Norm, selbst eine schlechte oder falsche Norm spricht noch dafür, sich normgemäß zu verhalten. Und auch das Umgekehrte gilt: Richtigkeit schafft keine Existenz. Man kann sich eine Norm denken, die es in der Gesellschaft, in der man lebt, faktisch nicht gibt, die zu etablieren aber richtig wäre. Aber dass es richtig wäre, bedeutet eben nicht, dass die Norm existiert. Eine Norm und das normgenerierte Müssen sind soziale Phänomene. Sie kommen nicht dadurch in die Existenz, dass es richtig wäre, sie zu haben.

Versteht man das moralische Müssen hingegen „einfach“ hypothetisch, ergibt sich dieser Unterschied nicht. Wenn man einen Marathon laufen will und dafür trainieren muss, existiert ein normatives Müssen, aber die Frage, ob hier das Richtige der Gegenstand des Müssens ist, stellt sich nicht. Man würde die Frage gar nicht verstehen. Genauso, wenn man sich moralisch verhalten muss dafür, dass es zu nützlichen Kooperationen kommt. Wenn man solche Kooperationen anstrebt, besteht ein normatives „Muss“, sich moralisch zu verhalten. Aber auch in diesem Fall würde man die Frage, ob hier das Richtige der Gegenstand des Müssens ist, gar nicht verstehen. Die Differenz von Existenz und Richtigkeit ergibt sich naheliegenderweise nur, wenn das moralische Müssen gemacht ist, wenn bestimmte Handlungen künstlich zu moralisch „gemussten“ gemacht werden. Dann stellt sich klarerweise die Frage, ob die moralischen Normen einer Gesellschaft das Richtige zum Gegenstand ihres Müssens machen.

Es fällt auf, dass viele Autoren Schwierigkeiten haben, diesen Unterschied von Existenz und Richtigkeit zu verstehen. Darin spiegelt sich, dass sie das moralische Müssen nicht als sanktionskonstituiertes, soziales Phänomen verstehen, 
sondern bewusst oder unbewusst als vorsozial, entweder als „einfaches“ hypothetisches Müssen oder als kategorisches.

Es scheint, als versperre außerdem eine von Kant herkommende Vorstellung den Zugang zu dieser Unterscheidung. Oft wird gesagt, dass wir moralisch handeln müssen, sei ein „Vernunftgebot“. Natürlich ist das nur eine Metapher - die Vernunft ist keine Person, die etwas gebietet. Man hat indes keine Mühe, zu verstehen, was gemeint ist. Man muss verschiedene Dinge, das ist die einfache Tatsache, um die es geht, vernünftigerweise tun. Wenn ich die Meisterprüfung bestehen will, muss ich vernünftiger- oder überlegterweise die vorbereitenden Kurse besuchen. Stattdessen kann man dann - etwas pompös - auch sagen, die Vernunft gebiete mir, die Kurse zu besuchen, oder es sei ein Vernunftgebot, sie zu besuchen.

Nimmt man die Metapher allzu wörtlich, kommt es schnell zu der Vorstellung, ein Vernunftgebot komme aus der Vernunft und sei damit etwas ganz anderes als eine soziale Norm, hinter der die Gesellschaft steht. So entsteht ein Gegensatz von Vernunftgebot und sozialem Gebot, und wenn man die Moral als ein Set von „Vernunftgeboten“ versteht, transportiert man damit ein vorsoziales Verständnis des Moralischen. Zudem scheint klar, dass sich, wenn es die Vernunft ist, die etwas gebietet, keine weitere Frage stellt. Wo sollte hier noch Platz sein für die Frage nach der Richtigkeit des Gebots?

Doch mit all dem verfängt man sich nur in den Fallstricken der Metapher. Denn wenn es aufgrund einer sanktionskonstituierten, sozialen Norm ein „Muss“ ist, die Handlung $x$ zu tun, und man folglich, wenn man es doch nicht tut, sanktioniert wird, dann ist es, wenn man sich dieser Metapher bedienen will, ein Vernunftgebot, $x$ zu tun. Es gibt offenkundig einen Grund, $x$ zu tun. Ein Gegensatz von sozialer Norm und Vernunftgebot besteht also in Wirklichkeit nicht. Eine Norm ist oder schafft ein Vernunftgebot. Und natürlich stellt sich in diesem Fall auch die Frage, ob die Norm das Richtige gebietet und ob es nicht besser wäre, wenn es nicht geboten wäre, $x$ zu tun. Diese Frage wird durch die Rede vom Vernunftgebot keineswegs überflüssig. Man sollte sich durch die Metapher, so zeigt diese Überlegung, nicht unbemerkt in ein vorsoziales Verständnis des moralischen Müssens hineinziehen lassen. Am besten wäre es, die irreführende Rede vom Vernunftgebot ganz fallen zu lassen.

\section{5}

In diesem Zusammenhang ist ein weiterer Punkt wichtig. Ein „einfaches“ hypothetisches Müssen erlaubt es nicht (außer in der Form der Metapher), die Begriffe „geboten“, „verboten“ und „Pflicht“ einzuführen. Wenn ich trainieren muss, weil 
ich einen Marathon laufen will, dann gibt es kein Gebot, dies zu tun, es gebietet niemand. Es ist einfach notwendig, es zu tun. Genauso, wenn man sich moralisch verhalten muss, um zu gewünschten Kooperationen zu kommen. Von Geboten und Verboten kann man offenkundig nur sprechen, wenn Personen etwas von einem verlangen. Die Begriffe setzen einen personalen Kontext voraus. Wenn man das moralische Müssen sozial versteht, ist dieser personale Kontext gegeben: Die anderen, mit denen man zusammenlebt, verlangen von einem, dass man sich so-und-so verhält, und man selbst verlangt es von ihnen. Und deshalb ist es angebracht, zu sagen, eine Handlung sei moralisch geboten oder verboten. Außerhalb dieses personalen Kontextes gibt es für diese Begriffe keine Basis; man kann sie dann allenfalls metaphorisch verwenden.

Dasselbe gilt für den Begriff der Pflicht. Wenn ich jetzt aufbrechen muss, weil ich den Zug nach Urnäsch erreichen will, bin ich nicht verpflichtet, dies zu tun. Und auch wenn ich moralisch handeln muss, weil ich dadurch glücklich werde oder sonst etwas erreiche, was ich will, handelt es sich um ein einfaches hypothetisches Müssen. Auch in diesem Fall hat der Pflichtbegriff keinen Platz. Es ist deswegen keine Überraschung, dass es den Begriff im antiken Eudaimonismus, wie ihn Platon und Aristoteles entwickelt haben, nicht gibt. Er hat in diesem Rahmen keine Funktion. Es ist immer wieder ganz zu Recht darauf hingewiesen worden, dass der Begriff der Pflicht, ebenso wie das Geboten- und Verbotensein, in einem personalen Kontext steht. ${ }^{14}$ Dieser Kontext ist, wie gesagt, gegeben, wenn das moralische Müssen ein soziales Müssen ist. Es ist die Gemeinschaft, die einen verpflichtet, sich in bestimmter Weise zu verhalten, und die mit Zurückweisung und Tadel reagiert, wenn man es nicht tut. Wobei, das ist wesentlich, nicht jede Norm verpflichtend ist. Verpflichtend ist nur eine Norm, die richtig ist. Eine Norm kann das Falsche verlangen. In diesem Fall existiert sie zwar, und sie schafft auch einen Handlungsgrund, aber sie ist nicht verpflichtend. ${ }^{15}$ Auch wenn man dies berücksichtigt, bleibt es dabei, dass es eine Pflicht nur geben kann, wo andere Menschen (oder übernatürliche Wesen) von einem ein bestimmtes Verhalten verlangen.

Versteht man die Moral als ein Ensemble einfacher hypothetischer Imperative, kann man also die Begriffe des Verboten- und Gebotenseins und der Pflicht nicht einführen. Sie haben keinen Ort. Und noch etwas folgt aus dem Gesagten: Etwas wird nicht sanktioniert, weil es verboten ist, es ist vielmehr verboten, weil es sanktioniert wird. Die Sanktionierung schafft erst das Verbotensein.

14 Vgl. z. B. Mill (1863), Kap. 5, Absatz 14; Adams (1999), 233; Wolf, S. (2009). 15 Vgl. hierzu Stemmer (2013a). 


\section{6}

Eine Moral, in der jetzt skizzierten Weise sozial-institutionell verstanden, kann nur funktionieren, wenn ihre Normen von den allermeisten derer, die mit ihr leben, bejaht und akzeptiert werden. Dies ist schon deshalb nötig, weil die Mitglieder der moralischen Gemeinschaft nicht nur die Adressaten der Moral sind, sondern zugleich auch ihre Autoren: Sie bringen sie dadurch, dass sie das nicht gewollte Verhalten sanktionieren, in die Existenz und machen sie effektiv. Es gibt in einer Moral keinen eigenen Stab, der dafür zuständig wäre. Deshalb ist eine Moral in anderer Weise auf die Zustimmung der Betroffenen angewiesen als eine Rechtsordnung. Dennoch können natürlich Einzelne bestimmte Normen ablehnen oder für Normen plädieren, die es faktisch nicht gibt, die es ihrer Einschätzung nach aber geben sollte.

Dies vor Augen wird eingewandt, die soziale Moral könne nicht alles sein, was wir unter einer Moral verstehen. Diesseits der faktischen, sozial etablierten Moral gebe es, wie die Möglichkeit der Kritik zeige, eine „persönliche Moral“, an der man die soziale Moral messe. ${ }^{16}$ Mit „persönlicher Moral“ sind dabei nicht persönliche Maximen gemeint, die man sich nur für die eigene Person gibt, die aber nichts für andere besagen, sondern persönliche Regeln, von denen man meint, sie seien für alle die richtigen, und von denen man deshalb will, dass sie zu allgemein verbindlichen sozialen Normen werden. ${ }^{17}$

Man kann, worauf dieser Einwand zielt und welche Intuitionen ihn speisen, vielleicht auch in folgender Weise fassen: Wenn es zu solchen von der faktischen Moral abweichenden Einschätzungen kommt, muss es doch offenbar diesseits dieser Moral und unabhängig von ihr bereits moralische Urteile über moralisch richtig und moralisch falsch geben. Also gibt es offenkundig so etwas wie eine Moral vor der sozialen Moral. Und die soziale Moral und ihre Sanktionen haben dann nur die Funktion, diese vorsoziale Moral zu verstärken. Worum es eigentlich geht und was eigentlich zählt, ist, so die Vorstellung, diese vorsoziale Moral, sie ist der Maßstab für die soziale Moral, nicht nur da, wo man diese kritisiert, sondern auch da, wo man sie gutheißt.

Um über diesen Einwand zu einem Urteil zu kommen, muss man zunächst fragen, wodurch eine moralische Norm richtig ist und wodurch falsch oder schlecht. Eine Norm kann schlecht sein, weil sie auf kognitiven Annahmen beruht, die falsch oder unbegründet sind und die man deshalb nicht machen sollte, etwa auf unhaltbaren religiösen oder metaphysischen Annahmen. Es ist

16 So Wolf, U. (1984), 47-59 u. 201.

17 Ebd., 53. 
klar, dass wir mit solchen moralischen Normen nicht leben wollen. Jeder, der einsieht, dass eine Norm in dieser Weise falsch ist, wünscht sich, sie los zu sein.

Was folgt aus einer solchen Kritik für unsere moralischen Urteile? Angenommen, jemand verletzt eine in dieser Weise falsche Norm. Was er tut, ist moralisch verboten und wird sanktioniert. Daran ändert sich durch die kognitive Kritik, solange nur Einzelne so denken, nichts. Man muss sich also normkonform verhalten. Dieses moralische Müssen existiert, es ist eine soziale Realität. Aus der Sicht dessen, der die Norm für falsch hält, ist an der Handlung jedoch moralisch nichts zu kritisieren. Sie ist moralisch in Ordnung. Er wird sich deshalb auch, soweit wie möglich, nicht an der Sanktionspraxis beteiligen. Die beiden Urteile, dass eine solche Handlung moralisch verboten ist und man sie unterlassen muss, und dass sie dennoch moralisch in Ordnung ist, können offenbar ohne Weiteres nebeneinanderstehen. Man kann stattdessen auch einfach sagen: Sie ist moralisch verboten, aber sie sollte es nicht sein.

Die kognitive Kritik stützt sich auf Standards für die Wahrheit und das Begründetsein kognitiver Annahmen sowie den Wunsch, nicht mit oder unter Normen zu leben, die auf Annahmen beruhen, die diesen Standards nicht genügen. In diese Kritik geht also keine vorgängige moralische Ressource ein, wenngleich sie den Effekt hat, dass man etwas, was faktisch moralisch verboten ist, für moralisch in Ordnung hält.

Man kann eine Norm auch deshalb schlecht finden, weil sie im Unterschied $\mathrm{zu}$ anderen nicht den eigenen Interessen entspricht. Man wünscht sich folglich eine Moral ohne diese Norm. Hierbei handelt es sich wohl nur um eine Ablehnung, nicht um eine Kritik der fraglichen Norm. In jedem Fall geht offenkundig auch in sie keine vorgängige moralische Ressource ein. Genauso ist es, wenn man für eine zusätzliche Norm plädiert, die es faktisch nicht gibt, weil es den eigenen Interessen entspräche, wenn es sie gäbe.

Eine moralische Norm kann auch dadurch schlecht sein, dass sie moralisch nicht in Ordnung ist. Wodurch ist sie das? Eine Norm, das ist der eigentliche, häufig vorausgesetzte, aber nur selten explizit formulierte Punkt, kann repressiv sein, das heißt, sie entspricht nicht den Interessen aller Betroffenen, wird aber doch allen gegenüber durchgesetzt. Auch die, die sie nicht wollen und nicht akzeptieren, müssen sich normkonform verhalten. Die Durchsetzung der Norm auch ihnen gegenüber wird damit zu einem Akt bloßer Macht. Und das finden wir moralisch anstößig.

Worauf stützt sich dieses moralische Urteil? Eine Möglichkeit besteht darin, dass die sozial etablierte Moral ein Verbot enthält, andere zu unterdrücken, also anderen gegen ihren Willen etwas abzupressen. Es wird nur nicht gesehen, dass man das auch mit Normen und auch mit moralischen Normen tun kann, dass also auch die Durchsetzung einer moralischen Norm repressiv sein kann. Es 
wird, anders gesagt, nicht gesehen, dass das Unterdrückungsverbot nicht nur eine Norm unter anderen, sondern darüber hinaus auch eine Metanorm ist. Die Kritik wäre dann eine immanente Kritik, sie stützt sich auf eine bereits in der sozialen Moral vorhandene Norm. Und damit nicht auf eine davon unabhängige Ressource.

Lassen wir offen, ob es andere Formen der immanenten Kritik gibt, solche, die sich nicht auf die Repressivität einer Norm beziehen, und, wenn ja, welche das sein könnten. So oder so ändert sich, worauf es in unserem Kontext ankommt, nichts.

Was, wenn eine immanente Kritik nicht möglich ist und man stattdessen von außen ein Kriterium heranträgt, das besagt, eine moralische Norm müsse, damit sie selbst moralisch in Ordnung ist, die-und-die Bedingungen erfüllen? Man operiert dann mit einer externen Definition der Moral und entwickelt gegebenenfalls von dort die Kritik an einzelnen Normen der faktischen Moral. Wir können uns, um das zu erläutern, auf einige in diesem Zusammenhang aufschlussreiche Überlegungen von Rainer Forst beziehen. ${ }^{18}$ Moralische Normen müssen, so sein erster Schritt, rechtfertigbar sein; wer eine moralische Norm zur Geltung bringt und von anderen ein entsprechendes Handeln fordert, muss das ihnen gegenüber rechtfertigen können. Sie haben ein Recht darauf, sie haben ein grundlegendes Recht auf Rechtfertigung. Damit moralische Normen rechtfertigbar sind, müssen sie, so der zweite Schritt, bestimmten Kriterien genügen, bei Forst sind es Allgemeinheit und Wechselseitigkeit. Man kann dann prüfen, ob die faktischen Normen (oder auch bloß gedachte und erwogene) diesen Kriterien entsprechen, und sie, wenn nicht, anhand dieses Maßstabs kritisieren.

Das Erfordernis, dass moralische Normen gerechtfertigt sein müssen, ist in dieser Konzeption eindeutig ein moralisches Erfordernis. Wo kommt dieses moralische Müssen her? Forst fällt an diesem Punkt meines Erachtens (obwohl er das zu vermeiden sucht) in einen Objektivismus zurück. Es ist einfach ein Stück moralischer Objektivität, dass jeder Mensch dieses Recht auf Rechtfertigung hat und deshalb jeder verpflichtet ist, das, was er anderen gegenüber tut, zu rechtfertigen. Man sieht, so Forst, in einer „praktischen ,Wahrnehmung““, dass es so ist. ${ }^{19}$ Die der faktischen Moral vorgängige moralische Ressource, die hier ins Spiel kommt, ist also, so scheint es, eine objektive moralische Gegebenheit. Dieser Weg ist jedoch aus den genannten Gründen ausgeschlossen. Vielleicht will man stattdessen sagen, es sei ein kategorisches Vernunftgebot, sein Verhalten, soweit es andere betrifft, ihnen gegenüber $\mathrm{zu}$ rechtfertigen. Aber ganz gleichgültig, wie

18 Vgl. Forst (2007) und darin besonders den titelgebenden Aufsatz.

19 Ders. (2005), 496; vgl. auch ders. (2007), 63 u. 93. 
man das im Einzelnen ausbuchstabierte, mit der Idee des kategorischen Müssens geriete man, wie wir sahen, nur in eine andere Sackgasse.

Es bleibt deshalb nur, zu sagen, moralische Normen seien einfach dadurch definiert, dass sie gerechtfertigt sein müssen. Das „Muss“ ist dann kein moralisches mehr, sondern ein definitorisches. Man muss moralische Normen rechtfertigen können, ansonsten verdienen sie das Attribut „moralisch“ nicht. Natürlich kann man so definieren. Aber warum tut man es gerade in dieser Weise? Die naheliegende Antwort lautet: weil man meint, es sei einfach moralisch geboten, das, was man anderen gegenüber tut, rechtfertigen zu können. Damit ist man aber über einen Umweg wieder bei einem moralischen Erfordernis, und es wiederholen sich dieselben Fragen. Oder, das ist eine alternative Antwort, man definiert so, weil man will, dass das Verhalten der Menschen und dann auch die moralischen Normen, die sie anderen gegenüber zur Geltung bringen, diesem Maßstab entsprechen. Man will eine Moral, die diesem Kriterium entspricht. Und man will es so, weil man nicht unterdrückt werden will, generell nicht und auch nicht durch Normen. In diesem Fall misst man die existierende Moral an einem Wollen. Man definiert sie so, weil man sie so will. Und wenn sie der Definition nicht entspricht, dann entspricht sie nicht diesem Wollen. Auch diese Kritik kommt also nicht aus einer moralischen Ressource.

Insgesamt gelangen wir so zu dem Ergebnis, dass die Kritik und Ablehnung einzelner Normen der faktischen Moral entweder eine immanente Kritik ist oder eine Kritik, die aus einem Wollen kommt. Die kognitive Kritik kommt, wie wir sahen, aus einem Wollen. Man will nicht mit Normen leben, die auf falschen oder irrationalen kognitiven Prämissen beruhen. Die interessegeleitete Ablehnung von Normen kommt ohnehin aus einem Wollen. Und die Kritik von einer externen Definition aus kommt, wie gezeigt, auch aus einem Wollen. Die Kritik entspringt also in keinem Fall einer vorgängigen, der sozial etablierten Moral vorgelagerten Moral. Man überprüft und kritisiert die geltende Moral entweder, das ist das häufigste und zugleich effektivste Verfahren, an ihren eigenen Standards, oder man plädiert gegen die faktische Moral, weil man etwas anderes, etwas Besseres will. Eine weitere Möglichkeit gibt es, so meine ich, nicht.

Ich kann jetzt den Einwand gegen ein soziales Verständnis des moralischen Müssens, es gebe neben und vor der sozialen Moral eine persönliche Moral, die einem erst den Maßstab gibt, die soziale Moral zu beurteilen, zurückweisen. Diese vorsoziale Moral gibt es nicht. Die Moral ist ein soziales, durch soziale Sanktionen konstituiertes Phänomen. Und eine Handlung wird, wie schon gesagt, nicht sanktioniert, weil sie moralisch verboten ist, sie ist moralisch verboten, weil sie sanktioniert wird. Sanktioniert wird sie, weil man nicht will, dass sie getan wird. Deshalb macht man sie zu etwas Verbotenem. Weil man ich nicht und die anderen auch nicht - nicht gefoltert werden will, macht man, 
jemanden zu foltern, künstlich zu etwas Verbotenem. Das Nicht-Gewolltsein der betroffenen Handlungen ist also der Grund der Sanktionierung, nicht ihr Verbotensein..$^{20}$ Und deshalb bestimmt, was die Menschen wechselseitig voneinander wollen, die Inhalte der Moral. Wer es anders sieht, möge sagen, von welcher Art das den Sanktionen vorausgehende Verbotensein ist, wenn es, wie ich zu zeigen versuchte, nicht ein Stück moralischer Objektivität sein kann, wenn es nicht ein kategorisches Vernunft-Muss sein kann und wenn es auch nicht ein „einfaches“ hypothetisches Müssen sein kann. - All dies bedeutet, das sei, um Missverständnissen vorzubeugen, noch einmal hervorgehoben, selbstverständlich nicht, dass man die geltende Moral nicht kritisieren kann und nicht fragen kann, ob die faktischen Normen die richtigen sind. Und es bedeutet auch nicht, dass man die geltenden Normen nicht moralisch kritisieren kann. Wir tun es ständig und mit Recht: am Maßstab anderer, basaler Normen, die bereits Teil der faktisch existierenden Moral sind.

\section{7}

Auf einen weiteren Einwand will ich nur kurz eingehen. Wenn man eine Sanktionstheorie anziele, dürfe man, so wird gesagt, nicht an faktische Sanktionen denken, worauf es ankomme, sei die Richtigkeit möglicher Sanktionen. Eine Handlung sei dann moralisch nicht in Ordnung und dürfe nicht getan werden, wenn es richtig sei, sie zu sanktionieren. Und das kann sie auch sein, wenn sie faktisch nicht sanktioniert wird. ${ }^{21}$ „Wir wären“, so schreibt Norbert Anwander, „auch dann moralisch verpflichtet, Nothilfe zu leisten, wenn niemand auf die Idee gekommen wäre, ihre Unterlassung zu sanktionieren.“22 Denn auch dann wäre es richtig, zu sanktionieren. Aber warum muss man, auch wenn nicht sanktioniert wird, Nothilfe leisten? Was konstituiert diese vorgängige Norm? Was konstituiert den Handlungsdruck? Die Tatsache, dass es richtig ist, zu sanktionieren, konstituiert dieses Müssen nicht. Wie sollen bloß hypothetische Sanktionen dadurch, dass sie richtig sind, ein reales moralisches Müssen konstituieren?

20 Forst hat demgegenüber wiederholt betont, die Sanktionen folgten „dem Ge- und Verbotenen und nicht andersherum“. Die Sanktionen der sozialen Moral sind dann nur etwas Hinzukommendes, aber nicht das, was das wirkliche moralische Müssen konstituiert; vgl. ders. (2005), 495; ebenso ders. (2015), 39.

21 Vgl. Anwander (2013), 253-254 u. 257-258; auch Hacker (1973), 155-156.

22 Ebd., 253. 
Wir stoßen hier wieder auf den elementaren Unterschied zwischen der Existenz und der Richtigkeit einer Norm. Die faktische Sanktion schafft die Existenz, aber sie schafft nicht die Richtigkeit der Norm. Sowenig, wie Richtigkeit Existenz schafft und Falschheit Nicht-Existenz. Doch genau dies wird häufig angenommen. Man prüft eine bloß gedachte oder erwogene Norm an den Kriterien für die Richtigkeit einer Norm und glaubt, weil die Norm richtig wäre, wenn es sie gäbe, gebe es sie auch schon. Aber in Wahrheit muss man sie erst in die Existenz bringen: durch die Etablierung einer entsprechenden Sanktionspraxis. Eine gedachte Norm ist, gleichgültig, welche Eigenschaften sie hat, keine wirkliche Norm.

Bei Philosophen, die durch den Neukantianismus und seine Terminologie beeinflusst sind, spielt in diesem Zusammenhang oft der Begriff der Geltung eine ungute Rolle. Der Begriff kommt aus trüben Quellen (besonders bei Hermann Lotze) und trägt nur zur Unklarheit und Konfusion bei. ${ }^{23}$ Wenn wir ihn wie im Alltag gebrauchen, bedeutet, dass eine Norm gilt, nicht mehr als, dass es sie gibt. Damit ist über ihre Qualität und ihre Richtigkeit gar nichts gesagt. In der philosophischen Literatur changiert „Geltung“ hingegen häufig zwischen Existenz und Richtigkeit und wird oft primär im Sinne von Richtigkeit verwandt. Forst etwa sagt: Eine Norm ,,ist“ [...] durch ihre Geltung, und ob sie gerechtfertigt ist oder nicht, betrifft diese Geltung. " ${ }^{24}$ Geltung bedeutet hier offenbar Gerechtfertigtsein, und die Norm, die in diesem Sinne gilt, die ist auch, das heißt, diese Norm gibt es auch. Die Richtigkeit schafft also die Existenz. Doch tatsächlich schafft nicht die Richtigkeit eine Norm, faktische Sanktionen schaffen eine Norm. Sie schaffen aber nicht die Richtigkeit der Norm. Ob das Richtige sanktioniert wird, ist eine andere Frage. Man muss, wie gesagt, die Existenz und die Richtigkeit einer Norm auseinanderhalten.

\section{8}

Die moralischen Sanktionen reagieren, so habe ich gesagt, nicht auf das vorgängige Verbotensein der einschlägigen Handlungen, sondern auf ihr Nicht-Gewolltsein. Ich und auch die anderen wollen, dass bestimmte Handlungen unterlassen und andere getan werden. Deshalb wird, sich anders zu verhalten, sanktioniert, und dadurch werden die betreffenden Handlungen zu moralisch gebotenen und verbotenen Handlungen.

23 Vgl. Stemmer (2008), 187-192.

24 Forst (2010), 160. - Wie konfus die Rede von der „Geltung“ ist, zeigen z. B. H. Rickerts Ausführungen in Rickert (1921), 121-129. 
Das sei, so wird eingewandt, jedoch nur das Programm. Tatsächlich setzten die typischen moralischen Sanktionen das Verbotensein der Handlungen voraus. Anders seien sie gar nicht zu verstehen. ${ }^{25}$ Und deshalb enthalte die Sanktionstheorie einen Zirkel: Die Sanktionen sollen das Verbotensein erst konstituieren, gleichzeitig setzen sie es aber schon voraus.

Ob dies zutrifft, hängt offenkundig davon ab, von welcher Art die moralischen Sanktionen sind. Sie bestehen, so meine ich, in sozialem Wertverlust, im Verlust von Anerkennung und Akzeptanz, in sozialer Zurückweisung und Distanzierung in einer Vielzahl von Facetten und Steigerungsstufen. Sie machen sich einen der stärksten Wünsche der Menschen zunutze, den Wunsch nach Anerkennung. Auf dem Weg der Internalisierung wandern die äußeren Sanktionen nach innen und wandeln sich zu inneren Sanktionen. Man reagiert mit innerem Unbehagen und innerem Widerstand, wenn man selbst eine der gesellschaftlich geächteten Handlungen tut oder nur zu tun erwägt. Die inneren Reaktionen sind Abkömmlinge der äußeren Sanktionen, und deshalb scheint es trotz ihrer Spezifik gerechtfertigt, auch hier von Sanktionen zu sprechen. Sie treffen uns, weil wir nicht nur die Anerkennung durch andere wollen, sondern auch vor uns selbst bestehen wollen.

Mit all diesen Varianten der Zurückweisung reagiert man auf Verhaltensweisen, die die Menschen wechselseitig sehr stark nicht wollen, weil sonst etwas ins Rutschen kommt, worauf sie elementar angewiesen sind. Alle diese Reaktionsweisen kann man erklären, ohne auf ein vorgängiges Verbotensein rekurrieren zu müssen. So dass der Zirkeleinwand ins Leere läuft.

Der Einwand unterstellt offenbar andere moralische Sanktionen. Versuchen wir, uns das klarzumachen. Mit den Sanktionen, wie ich sie jetzt erläutert habe, entsteht die Moral, es entstehen moralische Normen und damit die Möglichkeit, Handlungen als moralisch und unmoralisch $\mathrm{zu}$ klassifizieren. Ist diese soziale Institution soweit etabliert, werden spezifisch moralische Gefühle möglich, die darauf reagieren, dass jemand, oder man selbst, etwas Unmoralisches getan hat. So reagiert man mit dem Gefühl der Empörung, der moralischen Gestalt des Zorns, auf ein moralisches Unrecht. Dieses und andere Gefühle gleicher Art setzen also das moralische Verbotensein als etwas von ihm Unabhängiges voraus. Der Zirkeleinwand wäre folglich gerechtfertigt, wenn die zentralen moralischen Sanktionen (so, wie es Tugendhat annimmt) die spezifisch moralischen Affekte wären. ${ }^{26}$ Das ist aber, wie ich meine, nicht der Fall. Die moralischen Gefühle, auf andere gerichtet oder auf einen selbst, sind höherstufige Phänomene, die in der

25 Vgl. z. B. Scarano (2006), 107-108; vgl. auch Birnbacher (2003), 9 ff.

26 Vgl. zu Scaranos Kritik an Tugendhat dessen Erwiderung in: Tugendhat (2006), $283 \mathrm{ff}$. 
Tat das moralische Verbotensein schon voraussetzen und es nicht konstituieren. Sie selbst sind keine Sanktionen, sondern spontane Reaktionen auf geschehenes Unrecht. ${ }^{27}$ Allerdings ist es, nicht anders als bei den Sanktionen, unangenehm, ihr Anlass und Gegenstand zu sein. Man will nicht, dass andere sich über einen empören, und will auch nicht, dass man sich über sich selbst empört. Die moralischen Gefühle sind außerdem auch Quellen weiteren sanktionierenden Verhaltens.

Ich will noch anmerken, dass sich die Eigenschaft des Verbotenseins, auch wenn es so ist, dass sie durch eine Sanktionspraxis entsteht, unweigerlich verselbstständigt und objektiviert. Man wächst in eine Gesellschaft hinein und lernt, dass bestimmte Handlungen moralisch verboten sind, und man spricht über sie, als sei das Verbotensein eine ihrer objektiven, von uns unabhängigen Eigenschaften. Tatsächlich ist es nicht einmal falsch, zu sagen, die Sanktionen reagierten auf verbotene Handlungen. Denn eine konkrete Handlung wird sanktioniert, weil Handlungen diesen Typs verboten sind. Es ist eine der Handlungen, die in der Gesellschaft als nicht akzeptabel gelten. Die nächste Frage, wodurch sie verboten sind, braucht man nicht zu stellen. Die Alltagsintuition wird deshalb immer wieder die Vorstellung entwickeln, es gebe ein vorgängiges Verbotensein und die Sanktionen reagierten darauf. Aber dabei kann man nicht stehen bleiben. Die philosophische Reflexion muss erklären, was dieses Verbotensein konstituiert und wie es in die Welt kommt.

\section{9}

Alle oder fast alle Kritiker der Sanktionstheorie bringen folgenden Einwand vor. Einige halten ihn offenbar für den zentralen Einwand. ${ }^{28}$ Nach der Sanktionstheorie, so sagen sie, sei eine unmoralische Handlung, wenn sie im Verborgenen getan werde und deshalb nicht sanktioniert werde, nicht verboten. Denn die Sanktion schaffe nach dieser Theorie ja erst das Verbotensein. Dass in einer solchen Situation kein Verbotensein vorliege, sei aber ganz kontraintuitiv. Und auch wenn eine unmoralische Handlung zwar sanktioniert werde, der, der sie tut, die Sanktion aber nicht fürchtet, sie für ihn also nichts Negatives ist, sei die Handlung nach der Sanktionstheorie offenbar nicht verboten. Denn wenn einem die Sanktion gleichgültig ist und man sie deshalb nicht vermeiden will, entsteht

27 So auch Stemmer (2000), 121 u. 151.

28 Vgl. besonders Brosow (2013), 102 ff.; Buddeberg/Vesper (2013a), 21-24; Freitag (2017). 
kein normatives Müssen und damit auch kein Verbotensein. Auch das sei ganz kontraintuitiv. ${ }^{29}$ Der Einwand zieht also bestimmte Sondersituationen heran, sie demonstrieren, so die Annahme, mehr als deutlich, dass es ein Verbotensein gibt, das von den Sanktionen unabhängig ist. Ich habe diesen Kritikpunkt bereits behandelt. ${ }^{30}$ Da er aber immer wieder vorgebracht wird, versuche ich, eine hoffentlich überzeugendere Antwort zu geben.

Zunächst ist darauf hinzuweisen, dass in einer Situation, in der man unbeobachtet etwas Unmoralisches tut und deshalb die äußeren Sanktionen nicht greifen, keineswegs alle Sanktionen wegfallen. Es gibt verschiedene Arten derivativer Sanktionen, zu ihnen gehören die inneren Sanktionen, außerdem besteht fast immer das Risiko, doch entdeckt und dann auch sanktioniert zu werden. Dieses Risiko allein ist schon etwas Negatives, man will es nicht, und daraus resultiert ein Müssen: Man muss, wenn man das Risiko nicht eingehen will, die betreffende Handlung unterlassen. Es ist eher unwahrscheinlich, dass auch diese derivativen Sanktionen ausfallen. Aber nehmen wir (um den Gedanken einschlägig, wenn auch wenig realistisch zu machen) an, dass tatsächlich alle Sanktionen ausfallen. Auch in diesen Fällen ist es so, dass die unmoralischen Handlungen im Verborgenen in dem Sinne verboten sind, dass es Handlungen von der Art sind, für die in dieser Gesellschaft eine Sanktionierung vorgesehen ist. Normen beziehen sich auf generische, nicht auf konkrete Handlungen. ${ }^{31}$ Und es gibt eine Norm, die Handlungen dieser Art verbietet. Ebendeshalb würden die unentdeckt bleibenden Handlungen, wenn sie entdeckt würden, sanktioniert. Wenn man eine Liste der Handlungen erstellen würde, die von der Gesellschaft verboten sind, also eine Art Moralgesetzbuch, stünden Handlungen dieser Art mit auf der Liste. Die moralische Gemeinschaft steht ihnen keineswegs indifferent gegenüber. Wer so handelt, tut etwas, was moralisch geächtet ist, - auch wenn er in einer Sondersituation aufgrund eines epistemischen Defizits faktisch nicht sanktioniert wird. Gerade deshalb reagiert man, wenn man eine solche Handlung tut oder zu tun erwägt, normalerweise mit einem starken Unbehagen und mit innerem Widerstand. Man weiß, dass man etwas tut, was moralisch nicht in Ordnung ist und was, würde es entdeckt, sanktioniert würde.

29 Engländer spricht in Bezug auf diese beiden Sonderfälle vom „Problem der Normexistenz bei fehlender Sanktionierung“ und vom „Problem der Normexistenz bei fehlendem Sanktionsvermeidungswillen“; vgl. Engländer (2013), 199-202.

30 Stemmer (2000), 162-191; ders. (2008), 176-181 u. 323-326; ders. (2010), 166-167.

31 Vgl. hierzu Austin (1955), 19. Ein Gesetz oder eine Regel, so sagt Austin hier, „obliges generally to acts or forbearances of a class“ (Hervorh. im Orig.). Genauso Brennan et al. (2013), 3. 
Genauso in der umgekehrten, auch durch ein epistemisches Defizit gekennzeichneten Situation: Wenn jemand nichts Unmoralisches getan hat, aber irrtümlich wegen eines Unrechts sanktioniert wird, hat er deshalb nichts Verbotenes getan. Er würde, wenn man nur wüsste, wie es war, nicht sanktioniert.

Auch ein Attentäter, der Menschen tötet und dabei auch sich selbst ${ }^{32}$, tut etwas, was moralisch geächtet ist. Er tut etwas Verbotenes, etwas, was auf der Liste der verbotenen Handlungen an erster Stelle steht. Und deshalb würde er, wenn eine solche Sanktionierung nicht durch seine Selbsttötung unmöglich wäre, sanktioniert. Die Annahme Frank Brosows, eine Sanktionstheorie, wie ich sie verteidige, könne nicht einmal ,das gezielte Töten Unschuldiger in allen denkbaren Situationen als moralisch inakzeptabel [...] brandmarken“33, geht weit an der Sache vorbei. Natürlich ist eine solche Handlung moralisch inakzeptabel, wir würden sie sanktionieren, wenn sich der Täter dem nicht durch seinen eigenen Tod entzogen hätte.

Auch der, der zwar wegen eines Unrechts sanktioniert wird, den die Sanktion aber nicht stört, tut offensichtlich etwas, was gegen eine Norm verstößt, die in der Gesellschaft gilt. Gerade deshalb wird er ja sanktioniert. Er tut etwas, was in der Gesellschaft verboten ist.

Man kann sich fragen, was hier eigentlich der Stein des Anstoßes ist. Die verschiedenen Sondersituationen, um die es jetzt geht, sind, was ihre normative Beschaffenheit angeht, sehr eindeutig. Wenn faktisch nicht sanktioniert wird und auch die derivativen Sanktionen ausfallen, existiert offenkundig kein sanktionskonstituierter Handlungsgrund. Das normative Müssen, das die Sanktion schaffen soll, existiert nicht. Dies kann niemand bestreiten. Und wenn jemand die Sanktion nicht fürchtet, existiert, obwohl faktisch sanktioniert wird, der sanktionskonstituierte Handlungsgrund ebenfalls nicht. Wo kein Wollen, da auch kein normatives Müssen. Dies kann ebenfalls niemand bestreiten. Man will aber, das ist der springende Punkt, obwohl der sanktionskonstituierte Handlungsgrund fehlt, sagen können, dass jemand, der im Verborgenen zum Beispiel jemanden verletzt, etwas Unmoralisches und etwas Verbotenes tut. Das kann man aber aus den genannten Gründen auch auf dem Boden der Sanktionstheorie ohne weiteres sagen. Und man kann auch sagen, dass derjenige gegen eine moralische Norm verstößt. Es gibt eine etablierte Sanktionspraxis, die sich gegen Handlungen dieser Art richtet, und dadurch die entsprechende Norm. Wenn es auch so ist, dass die Norm in diesem speziellen Fall aus Mangel an Wissen die intendierte Wirkung verfehlt. Sie erreicht in dieser Situation eine bestimmte Person nicht. Der Versuch, mit Hilfe sozialer

32 Auf diese Figur spitzt Brosow die Diskussion zu.

33 Brosow (2013), 104. 
Normen das Verhalten möglichst aller in möglichst allen einschlägigen Situationen $\mathrm{zu}$ beeinflussen, ist, das ist eine einfache Wahrheit, nicht immer erfolgreich. Wenn die Norm, wie im Fall des Verletzungsverbots, eine legitime Norm ist, kann man aus denselben Gründen auch sagen, dass die betreffende Person gegen eine Pflicht verstößt. Auch das ist ohne Zweifel richtig.

Damit, dass die Norm den, der im Verborgenen etwas Unmoralisches tut, nicht erreicht, entfällt, wie gesagt, für ihn ein Grund, die Handlung zu unterlassen. Die Norm versagt darin, für ihn einen Grund und ein normatives Müssen zu schaffen. Um das besonders hervorzuheben, kann man auch sagen, dass die Norm in dieser Situation für diese Person nicht existiert. ${ }^{34}$ Dies, obwohl die Norm in der Gesellschaft existiert und es deshalb richtig ist, zu sagen, dass die Person sich normwidrig verhält.

Um einem weiteren Missverständnis vorzubeugen, sei noch Folgendes hinzugefügt: Dass eine Handlung in dem jetzt erläuterten Sinne verboten und normwidrig ist, kann natürlich für jemanden ganz unabhängig von einer Sanktion, also etwa im Verborgenen, ein Grund sein, sie nicht zu tun. Dieser Grund ist dann aber nicht sanktionskonstituiert (obwohl er eine Sanktionspraxis voraussetzt). Er resultiert vielmehr daraus, dass die betreffende Person nichts Verbotenes tun will. Mit diesem Wollen kommt dann ein zusätzliches Element hinein, und damit auch ein zusätzlicher Grund anderer Art.

\section{0}

Häufig wird die Sanktionstheorie des moralischen Müssens so verstanden, als sei sie auf die These festgelegt, es gebe nur ein mögliches Motiv, sich moralisch zu verhalten: den Wunsch, die Sanktion zu vermeiden. Das ist ein merkwürdiges und besonders unerquickliches Missverständnis, weil diese Schlussfolgerung so offenkundig falsch ist. ${ }^{35}$ Die Sanktionstheorie ist eine Theorie darüber, was das

\footnotetext{
34 So habe ich es in Stemmer (2008), 177 u. 181, getan. Aus diesem Grund habe ich sogar gesagt (ebd., 181), eine solche Handlung sei „nicht wirklich verboten“. Dies hat viele irritiert und ist in der Tat eine irreführende Formulierung. Ich habe jetzt erläutert, in welchem Sinne die betreffenden Handlungen verboten sind und dass es richtig ist, hier vom Verbotensein zu sprechen. Dies zu tun, entspricht, wie es scheint, auch dem üblichen Sprachgebrauch. Ein konkreter Ladendiebstahl ist auch dann etwas Verbotenes, wenn er nicht entdeckt und deshalb nicht sanktioniert wird. Warum? Weil im Strafgesetzbuch steht, dass Diebstähle auf die-und-die Weise sanktioniert werden und dies, wenn sie entdeckt werden, auch tatsächlich geschieht.
}

35 Vgl. hierzu ebd., 320-323 u. 192-197; ders. (2010), 168. 
moralische Müssen konstituiert, oder, anders gesagt, wodurch dieses Müssen existiert, keine Theorie über die Motivation zur Moral und ihre verschiedenen Erscheinungsformen. Die Etablierung des moralischen Müssens durch eine Sanktionspraxis zielt natürlich darauf, zum moralischen Handeln zu motivieren. Und zwar dadurch, dass sie einen künstlichen Grund schafft, der dafür spricht, sich so zu verhalten. Aber das bedeutet keineswegs, dass es keine anderen Motive zum moralischen Handeln gibt. Es wäre ganz abwegig, das anzunehmen. Warum sollte es so sein? Es gibt andere Motive, normunabhängige und normabhängige. So kann man sich aus persönlicher Sympathie moralisch verhalten, aus Mitleid, weil man das Leid des anderen nicht erträgt, oder aufgrund eines Selbstbildes, zu dem es gehört, für andere da zu sein und auf andere Rücksicht zu nehmen, oder auch aus prudentiellen Gründen der Reziprozität. In diesen Fällen verlangen die moralischen Normen ein bestimmtes Verhalten, aber man verhält sich unabhängig davon aus anderen Motiven so. Gäbe es die moralischen Normen nicht, würde man sich nicht anders verhalten. Oder man handelt moralisch, weil es für einen selbstverständlich ist, die moralischen Normen zu befolgen, ganz unabhängig von den Sanktionen, die einen treffen würden, wenn man anders handelte. Oder weil man einsieht, wie nützlich die Institution der Moral ist, oder weil man sich als eine moralische Person versteht, zu deren Selbstverständnis es gehört, das zu tun, was die Moral verlangt. In diesen Fällen handelt man normabhängig moralisch, aber nicht aus Furcht vor den Sanktionen. ${ }^{36}$

Die spezielle Art der Motivation, die mit der Institution der Moral angezielt wird, wird einem klar, wenn man sich vor Augen führt, dass sie zwei spezifische Bedingungen erfüllen soll. Die Moral ist, wie wir sahen, ein System des Müssens, und etwas tun zu müssen, bedeutet, es tun zu müssen, unabhängig davon, ob man es will oder nicht will. ${ }^{37}$ So ist es bei jedem normativen Müssen: Man muss, wenn man wieder gesund werden will und es dafür notwendig ist, eine Gymnastik zu machen, die Gymnastik machen, gleichgültig, ob man sie, für sich genommen, will oder nicht. Genauso muss man ein Versprechen halten, gleichgültig, ob man es will oder nicht. Durch das moralische Müssen soll also gerade, das ist die erste Bedingung, ein Motiv zum moralischen Handeln geschaffen werden, das unabhängig davon ist, ob man das moralische Verhalten, für sich genommen, will oder nicht. Es soll ein Motiv geschaffen werden, das von diesem Wollen unabhängig ist. Es kommt dann zum Zuge, wenn man das Moralische, für sich genommen, gerade nicht tun will, die Motive dieser Art also entfallen oder nicht weit genug reichen oder wenn sie angesichts gegenläufiger Motive nicht stark genug sind.

36 Ich habe dies genauer entwickelt in ders. (2013c).

37 Vgl. oben 629. 
Vielleicht würden wir uns eine Welt wünschen, in der sich alle Menschen von sich aus, aus Selbstlosigkeit, aus Nächstenliebe, aus Anteilnahme am Ergehen anderer, oder weil es in anderer Weise ihren Wünschen entspricht, durchgängig und in ausreichendem Maße zugunsten anderer verhalten. Doch die Welt ist nicht so. Deshalb bedarf es der Institution der Moral; sie schafft einen Grund für das moralische Verhalten, der unabhängig davon ist, dass man das moralische Verhalten für sich genommen will. Dabei soll dieser Grund, und das ist die zweite Bedingung, einerseits möglichst stark sein und möglichst alle erreichen und andererseits möglichst wenig voraussetzen. So soll er gerade nicht von anspruchsvollen, nicht allgemein geteilten Glücksvorstellungen oder Selbstkonzeptionen abhängig sein. Vielmehr soll er an etwas anschließen, was man bei allen voraussetzen kann, also an ein möglichst basales, allgemein geteiltes Interesse. Um dieses Ziel zu erreichen, dockt die Moral an den elementaren, bei allen Menschen äußerst starken Wunsch nach Anerkennung an. Die Menschen wollen die Anerkennung durch andere. Die Moral macht sich das zunutze und macht das Moralisch-Handeln und das Moralisch-Sein zur Vorbedingung dieser Anerkennung und damit indirekt auch der Anerkennung durch sich selbst.

In anderer Form und speziellerer Zuspitzung wird derselbe Einwand auch so formuliert: Eine Sanktionstheorie könne nur ein pflichtgemäßes moralisches Handeln verständlich machen, aber nicht ein moralisches Handeln aus Pflicht oder um der Moral willen. ${ }^{38}$ Denn wenn man nur auf die moralische Norm schaut und die negativen Konsequenzen, mit denen man rechnen muss, wenn man normwidrig handelt, dann handelt man, so der Gedanke, bloß pflichtgemäß (vorausgesetzt, die Norm ist verpflichtend), aber nicht aus Pflicht. Man habe dann ein nur äußeres Verhältnis zur Moral, aber kein „inneres“ und handele aus den falschen Gründen moralisch.

Und was wäre ein Handeln aus Pflicht, das die Sanktionstheorie angeblich nicht verständlich machen kann? Lässt man die spezifisch kantische Einbettung dieses Gegensatzes beiseite und folgt stattdessen den zurückliegenden Ausführungen, kann man folgende Motivationsvarianten unterscheiden. In einer ersten Variante schaut man nicht einfach auf die Sanktionen, sondern prüft, ob auch das Richtige sanktioniert wird, nämlich die Handlungen, von denen alle wollen, dass sie getan bzw. unterlassen werden. Wenn man findet, dass es so ist, kann man diese Handlungen auch tun, weil es eben diese Handlungen sind. Es sind die Handlungen, auf die es im Zusammenleben der Menschen ankommt, die, die aus der Perspektive aller unbedingt getan bzw. nicht getan werden sollen. Handelt man aus diesem Beweggrund, spielen das Verbotensein und die Sank-

38 So zum Beispiel Anwander (2013), 272; Buddeberg/Vesper (2013a), 25-26. 
tionen in der Motivation keine Rolle. Man handelt jetzt nicht mehr aus „negativen“ Motiven, weil man äußere oder innere Widerstände vermeiden will, sondern weil man positiv etwas will, weil einem daran liegt, dass das Zusammenleben der Menschen gelingt. ${ }^{39}$

Diese Haltung kann sich dann in folgender Weise fortentwickeln. Wenn man wechselseitig will, dass bestimmte Handlungen getan bzw. unterlassen werden, dann will man wahrscheinlich auch, dass sie moralisch geboten bzw. verboten sind, und man findet die Moral gut und richtig, die genau die entsprechenden Verbote und Gebote enthält. Man will dann die Moral, sie ist eine nützliche und notwendige Institution, und man tut deshalb, was sie verlangt und wozu sie verpflichtet. Auch diese Motivation schaut nicht auf die Sanktionen. In ihr spielt zwar die Tatsache, dass die Moral diese Handlungen gebietet, eine Rolle, aber nicht die Furcht vor Sanktionen. Deshalb kann man sagen, dass, wer aus dieser Motivation handelt, das Moralische um der Moral willen tut oder, wenn man so will, „aus Pflicht“. Man weiß, wie wichtig die Moral ist, und man weiß, dass sie das Richtige gebietet, und deshalb will man sie stützen und mittragen.

Dies will man nur, wenn sie, wie gesagt, das Richtige gebietet, wenn ihre Gebote mit anderen Worten verpflichtend sind. Das ist eine Vorbedingung für diese Haltung. Damit ist die „deliberative Relevanz von Verpflichtungen“, die Titus Stahl einfordert ${ }^{40}$, gegeben. Die Tatsache, dass die moralischen Gebote verpflichtend sind, spielt hier eine entscheidende Rolle. Wären sie, zum Teil, repressiv, könnte die Haltung zur Moral nicht in dieser Weise ungebrochen positiv sein.

Eine nächste Motivationsstufe wird erreicht, wenn, eine Person zu sein, die in dieser Weise positiv zur Moral steht, zum Teil des Selbstverständnisses wird. Man handelt dann moralisch, weil das zur eigenen Person gehört. Verhielte man sich anders, hätte man das Gefühl, nicht mehr der zu sein, der man ist. Entsprechend erziehen wir Kinder so zur Moral, dass es ihnen selbstverständlich ist, so zu handeln, und dass es möglichst eng mit dem Bild, das sie von sich selbst haben, verbunden ist. Das Verhältnis zur Moral, das so entsteht, ist ein „inneres“. Die Beweggründe zum moralischen Handeln verändern sich damit noch einmal grundlegend. ${ }^{41}$

Natürlich gibt es diese verschiedenen Versionen der Motivation, und man könnte ihre Phänomenologie noch erheblich verfeinern. Aber warum sollte die Sanktionstheorie das leugnen? Warum sollte sie das nicht verständlich machen können? Das ist nicht zu sehen. Es gibt viele Motive zum moralischen Handeln

39 Vgl. zu dieser Unterscheidung von positiven und negativen Motiven Wolf, U. (2013), 44. 40 Vgl. Stahl (2013), 318.

41 Vgl. hierzu Stemmer (2000), 145 ff. 
und viele, die mit Sanktionen nichts zu tun haben. Niemand bestreitet das. Die Sanktionstheorie ist, wie gesagt, keine Theorie der Motivation und keine Theorie über die richtigen oder falschen, die willkommenen oder weniger willkommenen Motive zur Moral, sondern eine Theorie des moralischen Müssens. Und dieses Müssen hat eine sehr spezielle Funktion, es zielt darauf, auch denen, die andere, der Moral entgegenkommende Motive nicht mitbringen oder nicht in ausreichendem Maße mitbringen, einen Grund und damit ein Motiv zu geben, sich moralisch zu verhalten. Das moralische Handeln muss für alle vernünftig sein, auch für die, die, wie Kant gesagt hat, „gleichgültig gegen die Leiden anderer“ sind. ${ }^{42}$

Dem Einwand, die Sanktionstheorie übersehe oder leugne die motivationale und deliberative Relevanz der Tatsache, dass moralische Normen verpflichtend sind (oder sein können), liegt, zumindest bei Stahl ${ }^{43}$, eine Passage in Normativität zugrunde, in der ich betone, dass, wenn eine Norm verpflichtend ist, „dem normativen Müssen, das aus den Sanktionen kommt“, dadurch „nicht ein zweites, spezifisch verpflichtendes Müssen hinzugefügt“ wird. ${ }^{44}$ Das eine Müssen, durch Sanktionen konstituiert, hat nur eine bestimmte Eigenschaft, die, verpflichtend zu sein, und es hat diese Eigenschaft dadurch, dass die Sanktionierung legitim ist oder, anders gefasst, das Richtige sanktioniert wird. Es entsteht also, das ist die Stoßrichtung dieser Passage, dadurch, dass ein normatives Müssen verpflichtend ist, nicht ein zusätzliches, zweites Müssen. Man muss, so habe ich gesagt, „den Mythos einer eigenen, spezifisch verpflichtenden Normativität aufgeben“ “. ${ }^{45}$ All dies scheint mir richtig zu sein. Wie sollte die Tatsache, dass ein Müssen eine bestimmte Eigenschaft hat, ein zu diesem Müssen hinzukommendes, zweites Müssen hervorbringen? Dennoch ist die Tatsache, dass man eine Handlung nicht nur tun muss, sondern es auch eine Pflicht ist, sie zu tun, ganz und gar nicht unbedeutend. Wir haben schon gesehen, dass man eine positive Haltung zur Moral entwickeln kann, weil einem bewusst ist, wie nützlich sie ist, und dass eine solche Haltung davon abhängig ist, dass man die moralischen Normen für verpflichtend und nicht für repressiv hält.

Auch in zwei weiteren Hinsichten ist der Aspekt des Verpflichtetseins von Bedeutung. Wenn eine Norm repressiv und nicht verpflichtend ist, ich also einem bloßen Zwang unterworfen bin, dann will ich mich dieser Norm möglichst entziehen und nicht entsprechend handeln. Ich rebelliere gegen die Norm und würde sie gerne weghaben. Es mag sein, dass ich mich unter dem Strich dennoch norm-

42 Kant (1911), 398.

43 Stahl (2013), 320.

44 Stemmer (2008), 267.

45 Ebd., 267-268. - Vgl. zum Verständnis des Pflichtbegriffs auch ders. (2013a). 
konform verhalte, weil die Sanktion schwerer wiegt. Aber es gibt diesen Widerstand, den es nicht gibt, wenn die Norm verpflichtend ist. Und das ist ein wichtiger Unterschied.

Und zweitens verändert, dass eine Norm repressiv ist, die Sanktionen und die Haltung ihnen gegenüber. Wenn man die äußeren Sanktionen für nicht gerechtfertigt hält, hat das Auswirkungen auf die inneren Sanktionen. Sie fallen weg oder werden zumindest abgeschwächt. Auch hierin liegt ein wesentlicher Effekt.

Die „deliberative Relevanz von Verpflichtungen“ ist also in verschiedener Weise gegeben. Es ist offenkundig von erheblichem Gewicht, ob die Normen der Moral verpflichtend sind oder nicht.

\section{1}

Ich möchte mit zwei Bemerkungen schließen. Zunächst: Man kann das Gefühl haben, eine Theorie, wie ich sie jetzt erläutert und verteidigt habe, überschätze die Bedeutung von Sanktionen für die Moral und die Bedeutung des durch Sanktionen konstituierten Müssens. ${ }^{46}$ Das mag sein. Wir handeln, wie gesagt, aus einer Vielzahl von Motiven zugunsten anderer, die mit diesem Müssen nichts zu tun haben. Und wer wollte abschätzen, ein wie großer Teil unseres altruistischen Verhaltens aus diesen motivationalen Ressourcen kommt? Je mehr, desto besser. Aber keine Gesellschaft verlässt sich auf diese Ressourcen, darauf, dass die Menschen unmittelbar, etwa aus Mitleid, motiviert sind, sich altruistisch zu verhalten, oder dass sie es tun, weil sie einsehen, dass es im Blick auf ihre Ziele förderlich ist. Vielmehr üben alle Gesellschaften Druck aus, um die Wahrscheinlichkeit zu erhöhen, dass das geschieht, von dem alle wechselseitig wollen, dass es geschieht. Und damit, so meine ich, beginnt die Moral. Zwischen dem individuellen Altruismus auf der einen und der Rechtsordnung auf der anderen Seite existiert dieser Bereich des gesellschaftlich erzeugten künstlichen Müssens, das durch informelle Sanktionen geschaffen wird.

Man muss auch sehen, dass die sozialen Sanktionen und ihre Effekte in doppelter Weise unsichtbar werden. Die äußeren Sanktionen werden internalisiert, und man handelt dann ,aus sich heraus“ moralisch. Außerdem werden die Kinder von vorneherein entlang der gesellschaftlichen Normen moralisch sozialisiert, so dass sie dann ebenfalls „,aus sich heraus“ das Moralische tun. Die Sanktionen zielen geradezu darauf, sich auf diese Weise selbst überflüssig zu machen.

46 So Steinfath (2003), 94. 
Die Dependenz des Verhaltens von den äußeren Sanktionen ist dann gar nicht mehr zu sehen. Dennoch gibt es die Sanktionen, und man kann schnell mit ihnen Bekanntschaft machen. Aber im Wesentlichen bestimmen die inneren Impulse das Verhalten, ohne dass man sich ihrer Herkunft bewusst ist.

Die zweite Bemerkung: In den Diskussionen über die Sanktionstheorie wird häufig gesagt, sie oder einzelne ihrer Elemente deckten sich nicht mit unseren Intuitionen. ${ }^{47}$ Doch selbst wenn es so sein sollte, kann diese Diagnose nur der Ausgangspunkt einer Untersuchung, aber nicht schon ihr Endpunkt sein. Eine Sanktionstheorie wird meines Erachtens immer in Spannung stehen zu unseren vortheoretischen Intuitionen, weil wir eine Tendenz haben, Verbote zu objektivieren. Wir tendieren immer zu der nicht weiter reflektierten Meinung, das-unddas sei verboten, nicht durch uns, sondern einfach objektiv. Und weil es verboten sei, werde es sanktioniert und nicht umgekehrt. Das hängt, wie schon erläutert, vor allem damit zusammen, dass uns als Kindern gesagt wird, dies und jenes sei verboten. Das wird uns beigebracht wie das Bestehen einer objektiven Tatsache. Intuitionen haben eine Vorgeschichte und möglicherweise eine in verschiedener Weise problematische Vorgeschichte, und schon deshalb können sie nicht unbesehen als Kriterium der Wahrheit taugen.

Es gibt in unserem Nachdenken gewiss elementare Punkte, an denen man glaubt, jeder sehe, dass etwas so-und-so ist, etwas sei evidentermaßen so, aber jenseits dieses basalen Niveaus sind Intuitionen Vorurteile, die vielen, zum Teil über Generationen eben „,intuitiv“ einleuchten. Dieser gewissermaßen evolutionäre Erfolg gibt ihnen ihr Gewicht, und man sollte sie keineswegs leichtfertig beiseiteschieben. Aber sie können nicht das letzte Wort sein. In der philosophischen Theoriebildung können und müssen wir fragen, welche Gründe sich für sie anführen lassen. Hier kann es offenkundig nicht dabei bleiben, dass etwas ,intuitiv“ einleuchtet. Man muss sich bewusst machen, ein sehr schwerwiegender Befund, dass die jahrhundertelange Unterdrückung der Frauen, die Abwertung der nicht-europäischen Völker, die Sklaverei, die Disqualifizierung der Homosexualität, um nur dies zu nennen, durch die moralischen Intuitionen gedeckt waren.

Und welchen Wert haben Intuitionen in einem so komplizierten und von Meinungsverschiedenheiten geprägten Feld wie der Metaethik? Kant war der Meinung, es leuchte „aus der gemeinen Idee der Pflicht und der sittlichen Gesetze“ ein, dass moralische Gebote kategorisch seien. ${ }^{48}$ Aber das entbindet nicht davon, zu fragen, ob, erstens, diese Ausdeutung der „gemeinen“ Vorstellung der Pflicht

47 Vgl. nur Buddeberg/Vesper (2013a), 10, 18 u. 26.

48 Kant (1911), 389. 
richtig ist, und ob, zweitens und wichtiger, die Idee eines kategorischen moralischen Müssens expliziert und mit Gründen verteidigt werden kann. Wer sich die Grundzüge der kantischen Moralphilosophie vergegenwärtigt, erkennt, dass seine Theorie kategorischer Imperative Teil eines hochkomplexen argumentativen Baukörpers ist. Über ihre Wahrheit und Falschheit kann man nicht anhand von Intuitionen entscheiden. Moralphilosophische Fragen mit Hilfe von Intuitionen lösen $\mathrm{zu}$ wollen, und die Idee, die Moralphilosophie insgesamt ziele auf nichts anderes als auf das Ordnen und Systematisieren unserer Intuitionen, wirkt auf mich, als spreche daraus ein beklagenswerter philosophischer Kleinmut.

\section{Literatur}

Adams, R. M. (1999), Finite and Infinite Goods. A Framework for Ethics, Oxford.

Anwander, N. (2013), Die halbe Wahrheit der Sanktionstheorie nebst einem Ausblick auf die andere Hälfte, in: Buddeberg/Vesper (2013b), 249-290.

Austin, J. (1955), The Province of Jurisprudence [1832], hg. v. Hart, H. L. A., London.

Birnbacher, D. (2003), Analytische Einführung in die Ethik, Berlin.

Brennan, G., Eriksson, L., Goodin, R. E., u. Southwood, N. (2013), Explaining Norms, Oxford.

Brosow, F. (2013), Wie sanktioniert man Selbstmordattentäter? Ein humeanischer Gegenentwurf zu Stemmers Kontraktualismus, in: Buddeberg/Vesper (2013b), 95-124.

Buddeberg, E., u. Vesper, A. (2013a), Beruht Moral auf Sanktion? Eine Problemübersicht, in: dies. (2013b), 9-31.

Buddeberg, E., u. Vesper, A. (Hg.) (2013b), Moral und Sanktion, Frankfurt am Main.

Engländer, A. (2013), Norm und Sanktion - Kritische Anmerkungen zum Sanktionsmodell der Norm, in: Rechtswissenschaft 4, 193-207.

Forst, R. (2005), Zur Aufklärung der (Moral)philosophie, in: Deutsche Zeitschrift für Philosophie 53, 493-497.

Forst, R. (2007), Das Recht auf Rechtfertigung, Frankfurt am Main.

Forst, R. (2010), Die Reise nach Phantasia, in: Deutsche Zeitschrift für Philosophie 58, 157-161.

Forst, R. (2015), Kritik der rechtfertigenden Vernunft. Die Erklärung praktischer Normativität, in: ders., Normativität und Macht, Frankfurt am Main, 37-57.

Freitag, W. (2017), Norm und Sanktion, in: Deutsche Zeitschrift für Philosophie 65.3, 436-455.

Hacker, P. M. S. (1973), Sanction Theories of Duty, in: Simpson, A. W. B. (Hg.), Oxford Essays in Jurisprudence, Oxford, 131-170.

Kant, I. (1781/1787), Kritik der reinen Vernunft, Riga, A =1. Aufl., B = 2. Aufl.

Kant, I. (1911), Grundlegung zur Metaphysik der Sitten [1785], in: ders., Werke 4, hg. v. d. Königl. Preuß. Akad. d. Wiss., Berlin, 385-463.

Kant, I. (1912), Untersuchung über die Deutlichkeit der Grundsätze der natürlichen Theologie und der Moral [1764], in: ders., Werke 2, hg. v. d. Königl. Preuß. Akad. d. Wiss., Berlin, 273-301.

Kant, I. (1923), Rezension zu Schulz's Versuch einer Anleitung zur Sittenlehre [1783], in: ders., Werke 8, hg. v. d. Königl. Preuß. Akad. d. Wiss., Berlin, 9-14.

Mackie, J. L. (1977), Ethics, London. 
Mill, J. St. (1863), Utilitarianism.

Rickert, H. (1921), Allgemeine Grundlegung der Philosophie, Tübingen.

Scarano, N. (2006), Die Sanktionstheorie der Moral. Überlegungen zum formalen Begriff der Moral bei Ernst Tugendhat, in: ders. u. Suárez, M. (Hg.), Ernst Tugendhats Ethik, München, 91-114.

Seebaß, G. (2002), Die sanktionistische Theorie des Sollens, in: Leist, A. (Hg.), Moral als Vertrag?, Berlin, 155-198.

Stahl, T. (2013), Sanktion, zweitpersonale Adressierung und Autorität, in: Buddeberg/Vesper (2013b), 317-330.

Steinfath, H. (2003), Wir und Ich. Überlegungen zur Begründung moralischer Normen, in: Leist, A. (Hg.), Moral als Vertrag?, Berlin, 71-95.

Stemmer, P. (2000), Handeln zugunsten anderer, Berlin.

Stemmer, P. (2008), Normativität, Berlin.

Stemmer, P. (2010), Normativität, Ontologie, Gründe, in: Deutsche Zeitschrift für Philosophie 58, 161-169.

Stemmer, P. (2013a), Der Begriff der moralischen Pflicht [2003], in: ders. (2013b), 20-52.

Stemmer, P. (2013b), Begründen, Rechtfertigen und das Unterdrückungsverbot, Berlin.

Stemmer, P. (2013c), Moral, künstliche Gründe und moralische Motivation [2006], in: ders. (2013b), 96-109.

Stemmer, P. (2013d), Was geht voraus: das Wollen den Gründen oder die Gründe dem Wollen? [2012], in: ders. (2013b), 139-165.

Stemmer, P. (2016), Der Vorrang des Wollens. Eine Studie zur Anthropologie, Frankfurt am Main.

Strawson, P. F. (1974), Social Morality and Individual Ideal [1961], in: ders., Freedom and Resentment and Other Essays, London, 26-44.

Tugendhat, E. (2006), Erwiderungen, in: Scarano, N., u. Suárez, M. (Hg.), Ernst Tugendhats Ethik, München, 273-312.

Wolf, S. (2009), Moral Obligations and Social Commands, in: Newlands, S., u. Jorgensen, L. M. (Hg.), Metaphysics and the Good, Oxford, 343-367.

Wolf, U. (1984), Das Problem des moralischen Sollens, Berlin.

Wolf, U. (2013), Vom moralischen Sollen, in: Buddeberg/Vesper (2013b), 35-49. 